\title{
Many-body theory of magneto-elasticity in one dimension
}

\author{
O. Tsyplyatyev and P. Kopietz \\ Institut für Theoretische Physik, Goethe-Universität Frankfurt, \\ Max-von-Laue Strasse 1, 60438 Frankfurt am Main, Germany

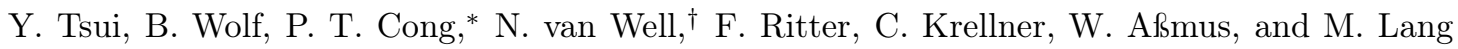
Physikalisches Institut, Goethe-Universität Frankfurt, Max-von-Laue-Strasse 1, 60438 Frankfurt am Main, Germany
\end{abstract}

(Dated: July 29, 2018)

\begin{abstract}
We construct a many-body theory of magneto-elasticity in one dimension and show that the dynamical correlation functions of the quantum magnet, connecting the spins with phonons, involve all energy scales. Accounting for all magnetic states non-perturbatively via the exact diagonalisation techniques of Bethe ansatz, we find that the renormalisation of the phonon velocity is a non-monotonous function of the external magnetic field and identify a new mechanism for attenuation of phonons - via hybridisation with the continuum of excitations at high energy. We conduct ultrasonic measurements on a high-quality single crystal of the frustrated spin- $1 / 2$ Heisenberg antiferromagnet $\mathrm{Cs}_{2} \mathrm{CuCl}_{4}$ in its nearly one-dimensional regime and confirm the theoretical predictions, demonstrating that ultrasound can be used as a powerful probe of strong correlations in one dimension.

PACS numbers: $73.63 . \mathrm{Nm}, 72.15 . \mathrm{Nj}, 75.45 .+\mathrm{j}, 72.55 .+\mathrm{s}$
\end{abstract}

\section{INTRODUCTION}

Magnetic insulators present a good example of interacting quantum systems where phonons can serve as an intrinsic probe of the strongly-correlated spins. $\frac{1}{2}$ The first microscopic theory of magneto-elasticity was developed at finite temperatures, $\stackrel{2,3}{\stackrel{3}{n}}$ where the static and the dynamic correlation functions of the spins were shown to couple to phonons with the same strength in the perturbative regime. At low temperature, assuming existence of a spin-liquid regime in two- and threedimensional Heisenberg antiferromagnets, phonons were shown to measure the mass and lifetime of the spin-liquid quasiparticles $, 4,5$ In one dimension (1D) - where interacting magnons form a spin-Luttinger liquid at low energy $\underline{\underline{6}}-$ the theory remains largely unexplored. At the same time such 1D systems are readily accessible in experiments

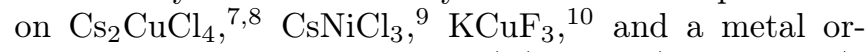
ganic coordination polymer $\mathrm{Cu}(\mathrm{II})-2,5$-bis(pyrazol-1-yl)1,4-dihydroxybenzene $\underline{11}$

In this paper we construct a microscopic theory of magneto-elasticity in $1 \mathrm{D}$ using the diagonalisation methods of Bethe ansatz: $\frac{12}{2}$ We derive the matrix elements for the four-point correlation function that couples the strongly-correlated spins to phonons dynamically and show that Luttinger liquid at low energy contributes comparably with the high-energy excitations that we are able to account for due to the hierarchy of modes $\underline{13}-\underline{15}$ The contribution of the static correlation function to the renormalisation of the sound velocity is parametrically larger than the dynamical correlation functions. The resonant decay of phonons in the many-body spin continuum vanishes very fast, as the fourth power of the length in large systems. However we identify another mechanism, hybridisation with the excitations at high energy via the dynamical correlation function, that remains finite in the thermodynamic limit. This work advances the many-body diagonalisation tools in $1 \mathrm{D}^{16-20}$ in to the field of magneto-elasticity, which is beginning to receive attention also in spintronics $\underline{21} \underline{\underline{24}}$

To test our theory we conduct ultrasonic measurements on a high-quality single crystal of $\mathrm{Cs}_{2} \mathrm{CuCl}_{4}$ in its nearly $1 \mathrm{D}$ regime, i.e. at temperatures of $0.7-2.1 \mathrm{~K}$ and magnetic fields up to $9 \mathrm{~T}: \stackrel{25,26}{2}$ The observed dependencies of the sound velocity and attenuation of the sound wave on the magnetic field agree well with all theoretical predictions. We find that the magnetic-field dependent part of the attenuation is governed by the hybridisation mechanism. Our results demonstrate that ultrasonic investigations, besides neutron-scattering experiments,,$\frac{7,10,27}{1}$ can be used as a powerful probe of correlation functions of the many-body system in $1 \mathrm{D}$ in magnetic insulators, just as tunnelling spectroscopy in semiconductor heterostructures $\stackrel{28,29}{ }$

The paper is organised as follows. Section II contains definition of the magnetostrictive interactions between the Heisenberg model and the phonon models in one dimensions and the diagonlisation of the isolated Heisenberg model by means of Bethe ansatz. In Section III we study renomalisation of sound velocity by evaluating microscopically the dynamical correlation function of the spins that couples to the phonons (Subsection IIIA) and by analysing it using the hierarchy of interacting modes (Subsection IIIB). In Section IV we consider different mechanisms of attenuation of phonons. And in Section V we conduct an ultrasound experiment on $\mathrm{Cs}_{2} \mathrm{CuCl}_{4}$ in its nearly one-dimensional regime and confirm the theoretical predictions. In Appendix A we derive the quantisaion equation for the pi-pairs' solutions of Bethe equations in the XY limit. In Appendix B we quote the normalisa- 
tion factor of the Bethe states together with the algebraic Bethe ansatz method. And in Appendix $\mathrm{C}$ we derive the matrix element of the spin operator needed for the magnetostrictive interaction.

\section{MODEL}

Theoretically, we consider phonons interacting with $1 / 2$-spins on a $1 \mathrm{D}$ lattice of length $L$ via a magnetostrictive interaction $\mathrm{as}^{2,3}$

$$
H=H_{m}+H_{p h}+V
$$

where

$$
\begin{aligned}
H_{m} & =\sum_{j=1}^{L}\left(J \mathbf{S}_{j} \cdot \mathbf{S}_{j+1}+B S_{j}^{z}\right), H_{p h}=\sum_{k} \omega_{k} a_{k}^{\dagger} a_{k}, \\
V & =\sum_{j=1}^{L}\left[J_{1}\left(x_{j+1}-x_{j}\right)+J_{2}\left(x_{j+1}-x_{j}\right)^{2}\right] \mathbf{S}_{j} \cdot \mathbf{S}_{j+1},
\end{aligned}
$$

are the Heisenberg model of spins, the free phonon model, and the interaction between them, respectively, $\mathbf{S}_{j}$ are the spin- $1 / 2$ operators, $J$ is the exchange interaction between spins when the atoms are in equilibrium, and $B$ is the external magnetic field in energy units. Here $a_{k}$ are Bose operators of the phonons, $\omega_{k}=2 \omega_{D}|\sin (k / 2)|$ is the phonon dispersion, $\omega_{D}$ is Debye energy, $x_{j}=$ $\sum_{k} \sqrt{\frac{\hbar b}{m v_{0}|k| L}}\left(a_{k}+a_{-k}^{\dagger}\right) e^{-i k j}$ is the position operator of an atom with the mass $m$ at lattice site $j$, and $v_{0}$ is the sound velocity. Phononic excitations modulate the exchange integrals resulting in a set of magnetostrictive constants $J_{n}=\left.\partial_{x}^{n} J(x)\right|_{x=b} / n$ ! that quantify the magneto-elastic interaction, where $b$ is the lattice parameter. We assume the periodic boundary condition: $\mathbf{S}_{j+L}=\mathbf{S}_{j}$ and $x_{j+L}=x_{j}$.

The spin Hamiltonian in Eq. (2) is diagonalised by $N$ magnon states parameterised with a set of $N$ quasimomenta $\mathbf{q}=\left(q_{1} \ldots q_{N}\right)$ that satisfy the non-linear Bethe equations 12

$$
q_{j} L-\sum_{l \neq j} \varphi_{j l}=2 \pi I_{j}
$$

where the two-body scattering phases are

$$
e^{i \varphi_{i j}}=-\frac{e^{i\left(q_{i}+q_{j}\right)}+1-2 \Delta e^{i q_{i}}}{e^{i\left(q_{i}+q_{j}\right)}+1-2 \Delta e^{i q_{j}}}
$$

$\Delta=1$, and $I_{j}$ is a set of non-equal integers. Solutions of Bethe equations can be found via numerical deformation from the XY point $\Delta=0$ (where $\varphi_{i j}=\pi$ gives the solutions $\left.q_{j}=2 \pi I_{j} / L\right)$ to the Heisenberg point $\Delta=$ 1.30 However, Bethe equations remain non-linear, $\alpha L-$ $\Phi(\alpha, \mathbf{q})=2 \pi I_{j}$, for some solutions that contain at least a pair of quasimomenta satisfying the condition $q_{i}+q_{j}=$ $\pm \pi$ in the $\Delta=0$ limit - see derivation in Appendix A. Here $q_{i}= \pm \pi-\alpha, q_{j}=\alpha$, the scattering phase

$$
e^{i \Phi(\alpha, \mathbf{q})}=-\frac{i \frac{2 \lambda}{L} \sum_{j=1}^{N-2 r} \frac{1-\sin q_{j} \sin \alpha}{\sin q_{j}-\sin \alpha}+e^{i \alpha}}{i \frac{2 \lambda}{L} \sum_{j=1}^{N-2 r} \frac{1-\sin q_{j} \sin \alpha}{\sin q_{j}-\sin \alpha}-e^{-i \alpha}}
$$

depends on another quasimomenta, $n$ is the number of such pi-pairs, and $\lambda=1$. Solutions for $\alpha$ can be obtained again via deformation from the $\lambda=0$ to the $\lambda=1$ point. The eigenenergy of $H_{m}$ corresponding to the state $\mathbf{q}$ is

$$
\varepsilon=\sum_{j=1}^{N}\left(J \cos q_{j}-J+B\right)+\left(\frac{J}{2}-B\right) \frac{L}{2}
$$

and the total momentum - preserved by the translational invariance - is $Q=\sum_{j=1}^{N} q_{j}$.

We consider renormalisation of phonons by spins via the magnetostrictive interaction $V$ perturbatively. The perturbation series for the eigenenergy of $H$ is

$$
E(k)=\varepsilon_{0}+\omega_{k}+\langle k|V| k\rangle+\sum_{\{\mathbf{k}, \mathbf{q}\}} \frac{|\langle\mathbf{k}, \mathbf{q}|V| k\rangle|^{2}}{\varepsilon_{0}+\omega_{k}-\varepsilon_{\mathbf{q}}-\omega_{\mathbf{k}}},
$$

where $\varepsilon_{0}$ is the ground state energy of $H_{m}, \omega_{\mathbf{k}}$ is an eigenenergy of $H_{p h}$ parameterised by $M$ momenta $\mathbf{k}=$ $\left(k_{1}, \cdots, k_{M}\right)$. The unperturbed state $|k\rangle=|k\rangle_{p h}|0\rangle_{m}$ is a direct product of a single phonon $|k\rangle_{p h}$ and the spin ground state $|0\rangle_{m}$ and $|\mathbf{k}, \mathbf{q}\rangle=|\mathbf{k}\rangle_{p h}|\mathbf{q}\rangle_{m}$ are the intermediate states.

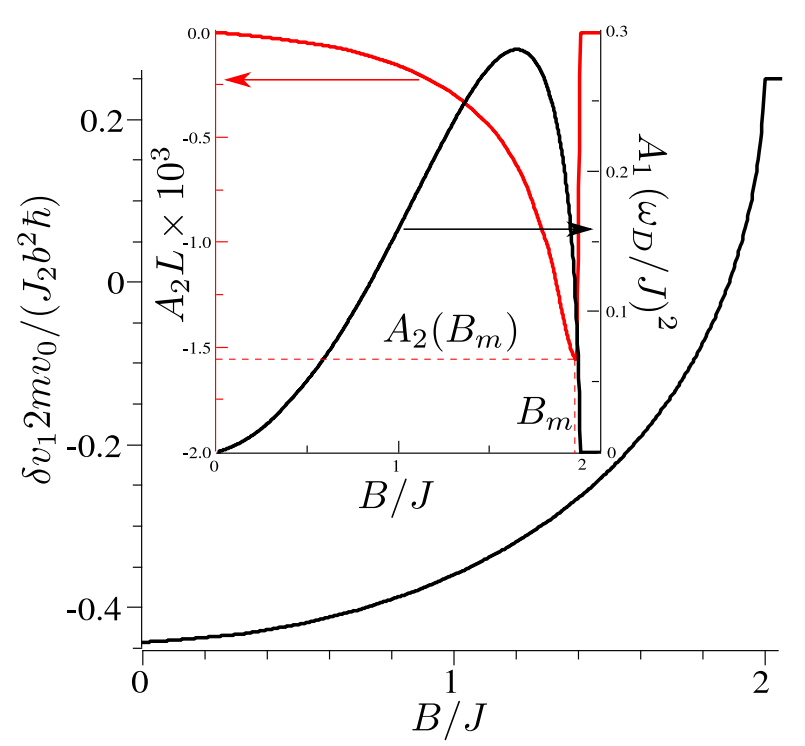

Figure 1. The static spin correlation function from Eq. (10) as a function of the magnetic field $B$, calculated using Bethe ansatz. Inset is the dynamic correlation function from Eq. (11) calculated using the matrix element in Eq. (13): the black line is the Luttinger liquid contribution in Eq. (20) and the red line is the high-energy contribution in Eq. (21). 


\section{RENORMALISATION OF SOUND VELOCITY}

Change of the sound velocity is given by a derivative of $E(k)$ as

$$
\delta v=\delta v_{1}+\delta v_{2}
$$

where evaluation of the phononic matrix elements leaves the spin correlation functions split into the static and the dynamic parts,

$$
\begin{aligned}
& \delta v_{1}=\frac{J_{2} b^{2} \hbar}{2 m v_{0}}\left\langle 0\left|\mathbf{S}_{1} \cdot \mathbf{S}_{2}\right| 0\right\rangle_{m} \\
& \delta v_{2}=\frac{J_{1}^{2} b^{2} \hbar}{m v_{0}} \sum_{\{\mathbf{q}\} ; Q=p} \frac{L\left|\left\langle\mathbf{q}\left|\mathbf{S}_{1} \cdot \mathbf{S}_{2}\right| 0\right\rangle_{m}\right|^{2}\left(\varepsilon_{0}-\varepsilon_{\mathbf{q}}\right)}{\left(\varepsilon_{0}-\varepsilon_{\mathbf{q}}\right)^{2}-\left(\omega_{D} p\right)^{2}}
\end{aligned}
$$

Here $\hbar p / b=2 \pi \hbar /(b L)$ is the quantum of the momentum and the sum over all of the many-magnon states, $\{\mathbf{q}\}$, is restricted by momentum conservation to the states with $Q=p$. The static correlation function in $\delta v_{1}$ is immediately obtained from $\varepsilon_{0}$ using the translational invariance $\operatorname{ass}^{31}$

$$
\left\langle 0\left|\mathbf{S}_{1} \cdot \mathbf{S}_{2}\right| 0\right\rangle_{m}=\frac{\varepsilon_{0}-B\left(N-\frac{L}{2}\right)}{J L} .
$$

Its dependence on the magnetic field, changing from the ferromagnetic value of 0.25 at high fields to the antiferromagnetic $\simeq-0.44$ in zero field, is shown in Fig. 1

\section{A. Dynamical correlation function of spins}

The dynamical part in $\delta v_{2}$ is a fourth-order correlation function. We evaluate the needed matrix element using the algebraic Bethe ansatz ${ }^{32}$ and obtain it as a sum over determinants of $N \times N$ matrices, see Appendix $\mathrm{C}$ for details,

$$
\begin{aligned}
& \left\langle\mathbf{q}\left|\mathbf{S}_{1} \cdot \mathbf{S}_{2}\right| 0\right\rangle=(\sqrt{\langle 0 \mid 0\rangle\langle\mathbf{q} \mid \mathbf{q}\rangle})^{-1}\left\{\frac{\prod_{i} \operatorname{ch}\left(v_{j}+\eta\right)}{\prod_{i<j} \operatorname{sh}\left(v_{i}-v_{j}\right)} \sum_{y}(-1)^{y} \frac{\prod_{i, j ; j \neq y} \operatorname{sh}\left(u_{j}-v_{i}\right)}{\prod_{j} \operatorname{ch}^{2}\left(u_{j}-\eta\right)} \prod_{l ; l \neq y} \frac{\operatorname{sh}\left(u_{l}-u_{y}+2 \eta\right)}{\operatorname{sh}\left(u_{l}-u_{y}\right)}\right. \\
& \left.\times\left[\operatorname{det} \hat{K}^{(y)}-\left(1-\frac{2 \operatorname{sh}(2 \eta) \operatorname{sh} \eta \operatorname{sh} u_{y} \prod_{j ; j \neq y} \operatorname{ch}\left(u_{j}+\eta\right)}{\prod_{i<j \neq y} \operatorname{sh}\left(u_{i}-u_{j}\right)}\right) \operatorname{det} \hat{G}^{(y)}\right]-\frac{\prod_{j} \operatorname{ch}\left(u_{j}+\eta\right) \prod_{j} \operatorname{ch}\left(v_{j}+\eta\right)}{\prod_{j} \operatorname{ch}^{2}\left(u_{j}-\eta\right) \prod_{i<j} \operatorname{sh}\left(v_{i}-v_{j}\right)} \operatorname{det} \hat{K}\right\},
\end{aligned}
$$

where the matrix elements are

$$
\begin{gathered}
K_{a b}=T_{a b}+\frac{(-1)^{b} \operatorname{sh}^{3}(2 \eta) \operatorname{sh} u_{b} \prod_{l ; l \neq b} \operatorname{sh}\left(u_{l}-u_{b}+2 \eta\right)}{\prod_{i<j \neq b} \operatorname{sh}\left(u_{i}-u_{j}\right) \prod_{l ; l \neq b} \operatorname{sh}\left(u_{l}-u_{b}\right)} \frac{\operatorname{sh} \prod_{j, i ; i \neq b} \operatorname{sh}\left(u_{i}-v_{j}\right)\left[\frac{\operatorname{sh} u_{b}}{\operatorname{ch} \eta}+\sum_{l} \frac{\operatorname{sh}(2 \eta) \operatorname{ch}\left(u_{b}+\eta\right)}{\operatorname{ch}\left(v_{l}-\eta\right) \operatorname{ch}\left(v_{l}+\eta\right)}\right]}{\operatorname{ch}\left(u_{b}+\eta\right) \operatorname{ch}\left(u_{b}+\eta\right) \operatorname{ch}\left(v_{a}-\eta\right) \operatorname{ch}\left(v_{a}+\eta\right)}, \\
K_{a b}^{(y)}=T_{a b}+\frac{(-1)^{b} \operatorname{sh}^{3}(2 \eta) \operatorname{sgn}(y-b) \operatorname{ch}\left(u_{y}-\eta\right)}{\operatorname{ch}\left(v_{a}-\eta\right) \operatorname{ch}\left(v_{a}+\eta\right) \prod_{i} \operatorname{sh}\left(u_{b}-v_{i}\right)} \frac{\operatorname{ch}\left(u_{b}+\eta\right) \prod_{i<j \neq y, b} \operatorname{sh}\left(u_{l}-u_{b}+2 \eta\right)}{\operatorname{sh}\left(u_{i}-u_{j}\right) \prod_{l ; l \neq y, b} \operatorname{sh}\left(u_{l}-u_{b}\right)} \\
\times\left[\frac{\operatorname{ch}\left(u_{b}-\eta\right)}{\operatorname{ch}\left(u_{b}+\eta\right)}-\frac{\operatorname{sh}\left(u_{y}-u_{b}+2 \eta\right)}{\operatorname{sh}\left(u_{y}-u_{b}-2 \eta\right)}+\frac{\operatorname{sh} 2 \eta \operatorname{ch}\left(u_{b}-2 \eta\right) \operatorname{sh} u_{y}}{\operatorname{ch}\left(u_{y}-\eta\right) \operatorname{ch}\left(u_{b}+\eta\right)}\right],
\end{gathered}
$$

when $b \neq y$ and

$$
K_{a y}^{(y)}=\frac{\operatorname{sh}(2 \eta) \operatorname{sh}\left(2 v_{a}\right)}{\operatorname{ch}^{2}\left(v_{a}-\eta\right) \operatorname{ch}^{2}\left(v_{a}+\eta\right)},
$$

$G_{a b}^{(y)}=T_{a b}$ when $b \neq y$ and $G_{a y}^{(y)}=K_{a y}^{(y)}$,

$$
T_{a b}=\frac{\operatorname{ch}^{L}\left(v_{b}-\eta\right)}{\operatorname{ch}^{L}\left(v_{b}+\eta\right)} \frac{\operatorname{sh}(2 \eta)}{\operatorname{sh}^{2}\left(v_{b}-u_{a}\right)} \prod_{j ; j \neq a} \frac{\operatorname{sh}\left(v_{b}-u_{j}+2 \eta\right)}{\operatorname{sh}\left(v_{b}-u_{j}\right)}-\frac{\operatorname{sh}(2 \eta)}{\operatorname{sh}^{2}\left(u_{a}-v_{b}\right)} \prod_{j ; j \neq a} \frac{\operatorname{sh}\left(u_{j}-v_{b}+2 \eta\right)}{\operatorname{sh}\left(u_{j}-v_{b}\right)} .
$$

The normalisation factors of Bethe states $33,34\langle 0 \mid 0\rangle$ and $\langle\mathbf{q} \mid \mathbf{q}\rangle$ are quoted in Appendix B in terms of a determinant of an $N \times N$ matrix. Here $\eta=(\operatorname{acosh} 1) / 2$,

$$
u_{j}=\ln \left(\frac{\sqrt{1-e^{i q_{j}^{0}-2 \eta}}}{\sqrt{1-e^{-i q_{j}^{0}-2 \eta}}}\right)-i \frac{q_{j}^{0}}{2}
$$


are the quasimomenta of the ground state $\mathbf{q}^{0}$ in Orbach parametrisation, and $v_{j}$ is obtained from $u_{j}$ by $q_{j}^{0} \rightarrow q_{j}$ where $\mathbf{q}$ are the excited states $\underline{12}$

\section{B. Hierarchy of modes}

The excitations in the sum over $\mathbf{q}$ in Eq. (11) have the same number of quasimomenta as the ground state at a given magnetic field. They are constructed by removing a quasimomentum from the ground state distribution and promoting it to an empty position, see sketch in Fig. 2 We will label these excitations as $\psi \psi^{*}$-pairs.

The whole dynamical correlation function in Eq. (11) exhibits a hierarchy of modes governed by their spectral strength. $\underline{13}-15$ The excitations split into groups according to $n=1,2,3, \ldots \psi \psi^{*}$-pairs that have progressively smaller amplitudes of their matrix elements, $\left|\left\langle\mathbf{q}\left|\mathbf{S}_{1} \cdot \mathbf{S}_{2}\right| 0\right\rangle\right|^{2} \sim 1 / L^{2 n}$. We keep the first three levels of the hierarchy,

$$
\delta v_{2}=\frac{J_{1}^{2} b^{2} \hbar}{m v_{0} J}\left(A_{1}+A_{2}+A_{3}\right) .
$$

The first level consists of only one pair with the minimally possible momentum $Q=p$,

$$
A_{1}(B)=\frac{v_{m} J L^{2}\left|\left\langle p\left|\mathbf{S}_{1} \cdot \mathbf{S}_{2}\right| 0\right\rangle_{m}\right|^{2}}{2 \pi \omega_{D}^{2}},
$$

where $v_{m}=\left(\varepsilon_{p}-\varepsilon_{0}\right) / p$ is the renormalised velocity of Luttinger liquid and we have used smallness of the exchange energy compared with Debye energy, $J / \omega_{D} \sim$ $10^{-3}$ for general material parameters $\frac{35}{3}$ The only matrix element in Eq. (20) can be obtained using the bosonic modes of Luttinger liquid, $\frac{6}{,}$ where the dispersion is almost linear. We, however, use a more general Bethe ansatz approach here. Solutions of Bethe equations give $v_{m}$ directly that, together with the matrix element in Eq. (13), gives the magnetic field dependence of $A_{1}(B)$ shown in the inset (right axis) in Fig. 1.

There are polynomially many states in the second and in the third levels of the hierarchy,

$$
A_{2(3)}(B)=\sum_{\{\mathbf{q}\} ; Q=p} \frac{L\left|\left\langle\mathbf{q}\left|\mathbf{S}_{1} \cdot \mathbf{S}_{2}\right| 0\right\rangle_{m}\right|^{2}}{\varepsilon_{\mathbf{q}}-\varepsilon_{0}},
$$

where the summand in Eq. (11) was expanded in a Taylor series in $\omega_{D} p /\left(\varepsilon_{0}-\varepsilon_{\boldsymbol{q}}\right) \ll 1$ since the sum over $\mathbf{q}$ accumulates dominantly at high energy. Contribution of the low-energy excitations (for which $\left(\varepsilon_{0}-\varepsilon_{\boldsymbol{q}}\right) / \omega_{D} p \ll 1$ ) has an additional small factor $J^{2} / \omega_{D}^{2}$, like in Eq. (20). At intermediate energies, $\left(\varepsilon_{0}-\varepsilon_{\boldsymbol{q}}\right) \simeq \omega_{D} p$, the perturbation theory for $E(k)$ becomes inapplicable since these magnetic excitations are in resonance with the acoustic phonon. However, the width of the anti-crossing 38 $\lesssim J_{1} \sqrt{\hbar b \omega_{D}^{2} /\left(m J^{3} L^{5}\right)}$ is much smaller than the manymagnon level spacing $J / L$ that is still in the Luttinger liquid regime. The non-perturbative contribution of these

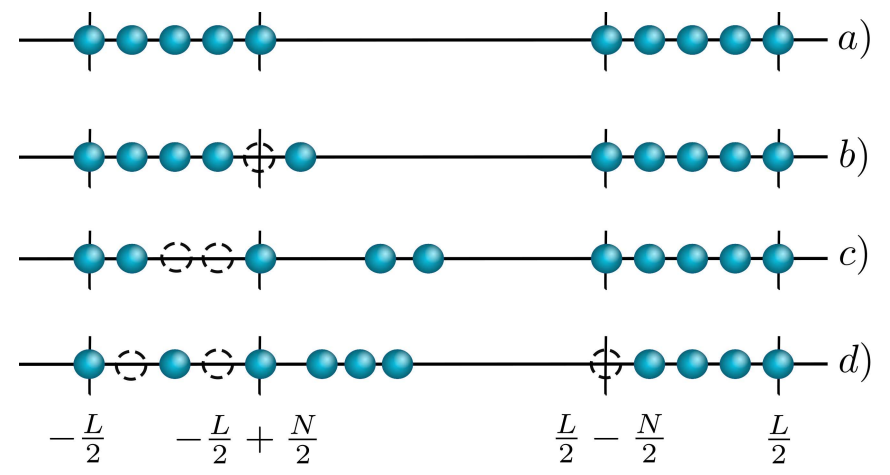

Figure 2. Configurations of non-equal integer numbers $I_{j}$ that correspond to the solutions the Bethe equations for the model $H_{m}$ : a) the ground state, b) one $\psi \psi^{*}$-pair excitation, c) two $\psi \psi^{*}$-pair excitations, b) three $\psi \psi^{*}$-pair excitations. These eigenstates include complex solutions at $\Delta=1$, which are obtained via numerical deformation of Bethe equations from the $\Delta=0$ to the $\Delta=1$ point.

levels is of the order of the anti-crossing width and vanishes in large systems.

We obtain the magnetic field dependence of $A_{2}$ numerically as a sum over the two $\psi \psi^{*}$-pairs in Eq. (21), see inset in Fig. 1. At high fields $A_{2}$ is small since there are only a few excitations, the strength of which is small as $1 / L^{4}$ at the second level of the hierarchy, and at small fields $A_{2}$ is again small since the majority of the excitations belongs to the class of pi-pairs close to the halffilling of the magnetic band, which makes their amplitudes even weaker than $1 / L^{4}$ due to Eq. (6). At the intermediate fields the $1 / L^{4}$ smallness is partially compensated by a large number of the excitations, whose majority does not have pi-pairs yet. The position of the maximum of $\left|A_{2}(B)\right|$ is identified from numerics at $B_{m}=2 J-9 \pi^{2} J /\left(2 L^{2}\right)$. The value of the function at this point is $A_{2}\left(B_{m}\right)=-0.0016 / L$ for large systems, see scaling of $A_{2}\left(B_{m}\right)$ in Fig. 3, which is small in a different parameter compared with $A_{1}$.

For typical values of material parameters, $A_{1}$ and $A_{2}$ are of the same order, e.g. $1 / L \sim 10^{-6}$ and $\left(\omega_{D} / J\right)^{2} \sim 10^{-6}$ for ultrasonic measurements in a magnetic insulator ${ }^{1}$. The three $\psi \psi^{*}$-pairs contribution $A_{3}$ is smaller than $A_{2}$ due to an additional $1 / L^{2}$ in accord with the hierarchy of modes $\underline{13}-15$ for the whole range of

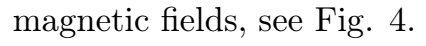

\section{ATTENUATION OF PHONONS}

Next let us analyse decay of the phonons into the spin excitations. The excitation spectrum of Heisenberg model in Eq. (2) is continuous which always has some states in resonance with the single phonon energy $\omega_{D} p$ providing a channel for the direct relaxation, unlike the previous phenomenological approaches $\frac{36,37}{\text { The rate of }}$ 


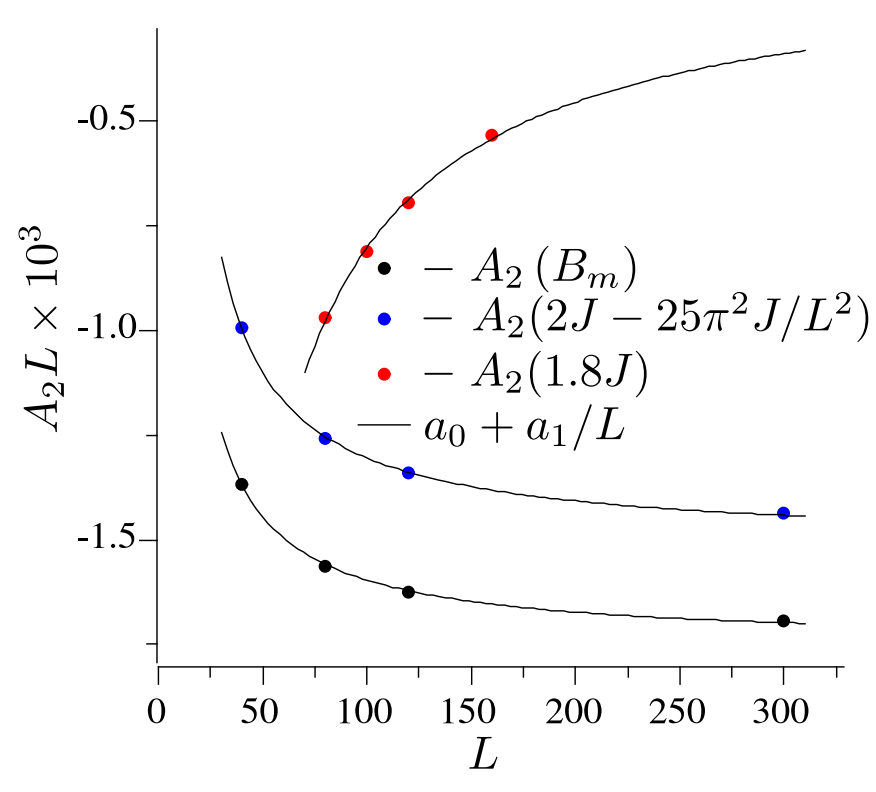

Figure 3. Scaling of $A_{2} L$ defined in Eq. (21) with the system length at three values of the magnetic field $B=B_{m}, 2 J-25 \pi^{2} J / L^{2}, 1.8 J$. The fitting of finite size corrections, $A_{2} L=a_{0}+a_{1} / L$, gives $\left(a_{0}, a_{1}\right) \times$ $10^{2}=(-0.17,1.51),(-0.15,2.04),(-0.01,-6.95)$ for the three magnetic fields respectively.

such a process is given by Fermi golden rule,

$$
\tau^{-1}=\frac{2 \pi^{2} J_{1}^{2} b}{m v_{0}} \sum_{\{\mathbf{q}\} ; Q=p}\left|\left\langle\mathbf{q}\left|\mathbf{S}_{1} \cdot \mathbf{S}_{2}\right| 0\right\rangle_{m}\right|^{2} \delta(\Delta E),
$$

where $\Delta E=\varepsilon_{\mathbf{q}}-\varepsilon_{0}-\omega_{p}$ and the contribution of the $J_{2}$ term in Eq. (3) is zero due to $\delta(\Delta E)$. The principal value of the sum in Eq. (22) is accumulated by the second level of the hierarchy, which we evaluate numerically see the magnetic field dependence of $\tau^{-1}$ in Fig. 5. Its maximum value has the same small prefactor $1 / L^{4}$ as the matrix element in Eq. (13) making the direct relaxation extremely slow in large systems.

However, the amplitude of the free phonons can also be reduced via hybridisation with the magnetic excitations, similarly to the $\delta v_{2}$ renormalisation of their velocity. The first order in perturbation theory for the wave function,

$$
\left|\Psi_{k}\right\rangle=|k\rangle+\sum_{\{\mathbf{k}, \mathbf{q}\}} \frac{\langle\mathbf{k}, \mathbf{q}|V| k\rangle}{\varepsilon_{0}+\omega_{k}-\varepsilon_{\mathbf{q}}-\omega_{\mathbf{k}}}|\mathbf{k}\rangle_{p h}|\mathbf{q}\rangle_{m},
$$

gives suppression at low momenta, $Z=1-\left|\left\langle p \mid \Psi_{p}\right\rangle\right|^{2}$, as

$$
Z=\frac{J_{1}^{2} b \pi \hbar^{2}}{m v_{0}} \sum_{\{\mathbf{q}\} ; Q=p} \frac{\left|\left\langle\mathbf{q}\left|\mathbf{S}_{1} \cdot \mathbf{S}_{2}\right| 0\right\rangle_{m}\right|^{2}}{\left(\varepsilon_{0}-\varepsilon_{\mathbf{q}}-\omega_{D} p\right)^{2}},
$$

which we analyse using the hierarchy of modes:

$$
Z=\frac{J_{1}^{2} b \pi \hbar^{2}}{J^{2} m v_{0}}\left(C_{1}+C_{2}+C_{3}\right)
$$

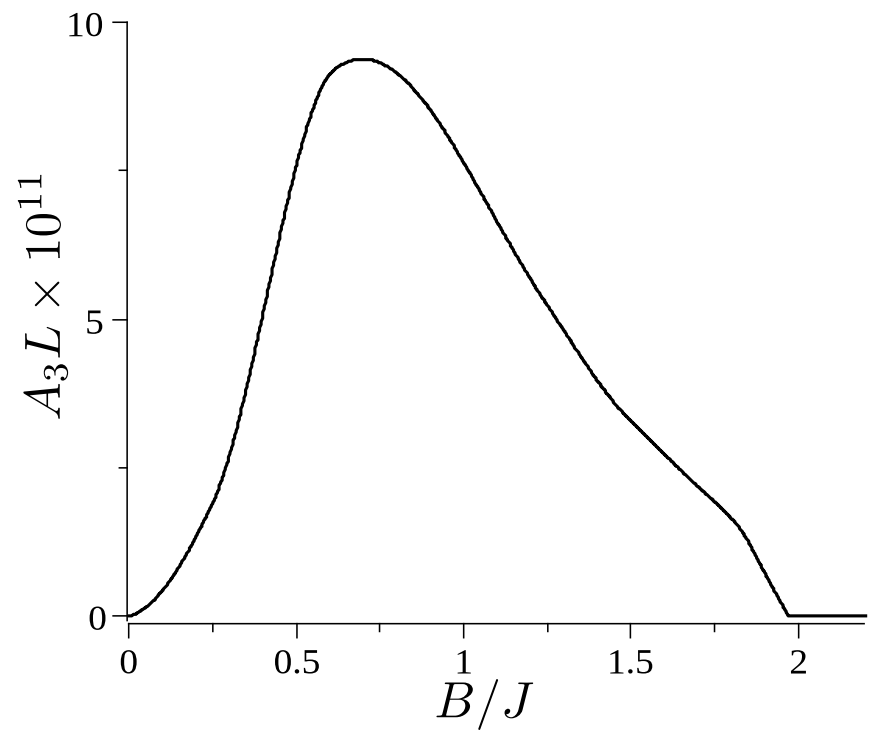

Figure 4. Contribution of the third level of the hierarchy of modes to $\delta v_{2}$ defined in Eq. (21); $L=40$. It is small compared with $A_{2}$ in inset in Fig. 1 for the whole range of magnetic fields.

where

$$
C_{1}=\frac{\left(J / \omega_{D}\right)^{2} L^{2}\left|\left\langle p\left|\mathbf{S}_{1} \cdot \mathbf{S}_{2}\right| 0\right\rangle_{m}\right|^{2}}{(2 \pi)^{2}}
$$

and

$$
C_{2(3)}=\sum_{\{\mathbf{q}\} ; Q=p} \frac{\left|\left\langle\mathbf{q}\left|\mathbf{S}_{1} \cdot \mathbf{S}_{2}\right| 0\right\rangle_{m}\right|^{2}}{\left(\varepsilon_{0}-\varepsilon_{\mathbf{q}}\right)^{2}},
$$

like in the analysis of Eq. (11) before. The first level contribution $C_{1}$ is small in $J^{2} / \omega_{D}^{2}$ like $A_{1}$, see inset in Fig. 5. But $C_{2}$, shown Fig. 5, remains finite in the thermodynamic limit (see scaling in Fig. 6) unlike $A_{2}$ above, and $C_{3}$ is small in $1 / L^{2}$ compared with $C_{2}$, see Fig. 7.

This hybridisation mechanism can be distinguished from other non-magnetic channels of relaxation via its magnetic field dependence and from the exponential decay into the resonant magnetic states described by Eq. (22) since it is constant in the temporal and spatial domains.

\section{ULTRASOUND EXPERIMENT ON $\mathrm{Cs}_{2} \mathrm{CuCl}_{4}$}

Finally, we discuss our experimental results. Highquality single crystals of several $\mathrm{mm}$ size of the frustrated spin- $1 / 2$ antiferromagnet $\mathrm{Cs}_{2} \mathrm{CuCl}_{4}$ were grown from an aqueous solution by an evaporation technique ${ }^{39} \mathrm{~A}$ pair of piezoelectric polymer-foil transducers was glued to opposite parallel surfaces perpendicular to the [010] direction for the generation and the detection of the ultrasound 


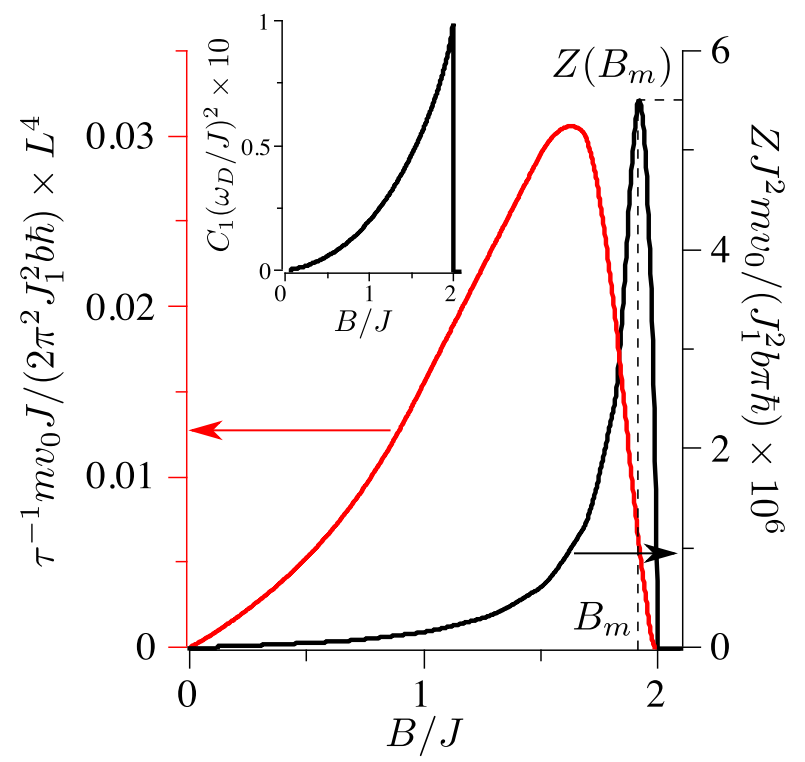

Figure 5. Two mechanisms of the sound attenuation: the red line is the relaxation rate $\tau^{-1}$ calculated using the Fermi golden rule in Eq. (22), while the black line is the degree of hybridisation $Z$ of a sound phonon with the magnetic excitations dominated by high energies in Eq. (24). Inset is the low energy contribution to Eq. (24).

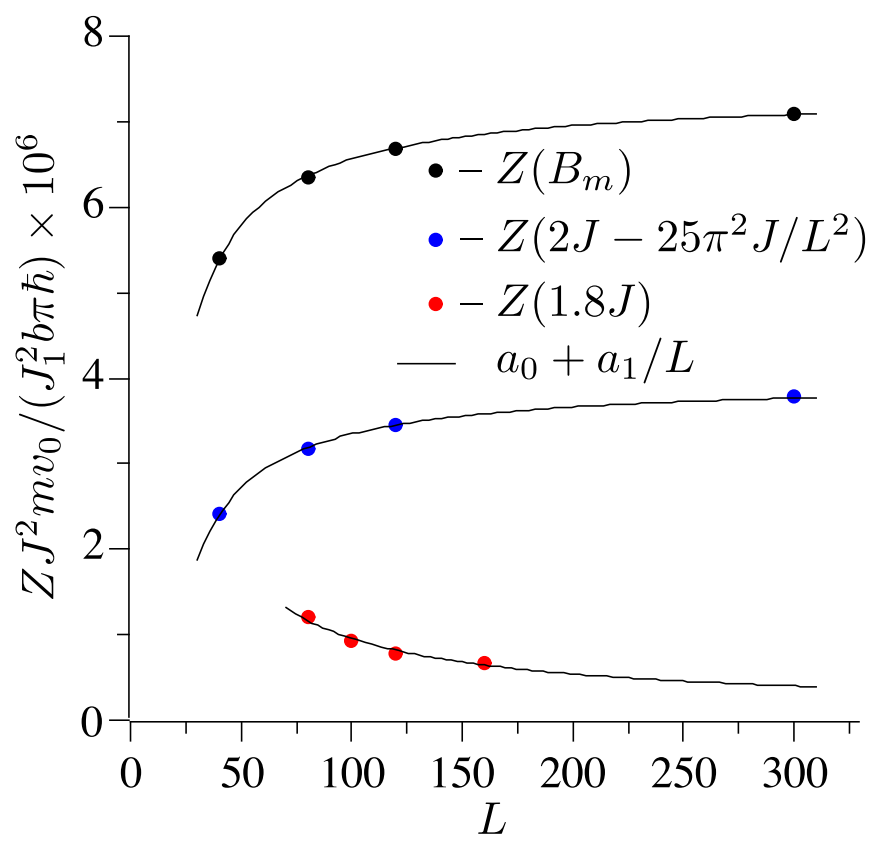

Figure 6. Scaling of $Z$ defined in Eq. (24) with the system length at three values of the magnetic field $B=$ $B_{m}, 2 J-25 \pi^{2} J / L^{2}, 1.8 J$. The fitting of finite size corrections, $Z J^{2} m v_{0} /\left(J_{1}^{2} b \pi \hbar^{2}\right)=a_{0}+a_{1} / L$, gives $\left(a_{0}, a_{1}\right) \times 10^{5}=$ $(0.73,-7.8),(0.40,-6.3),(0.012,8.4)$ for the three magnetic fields respectively.

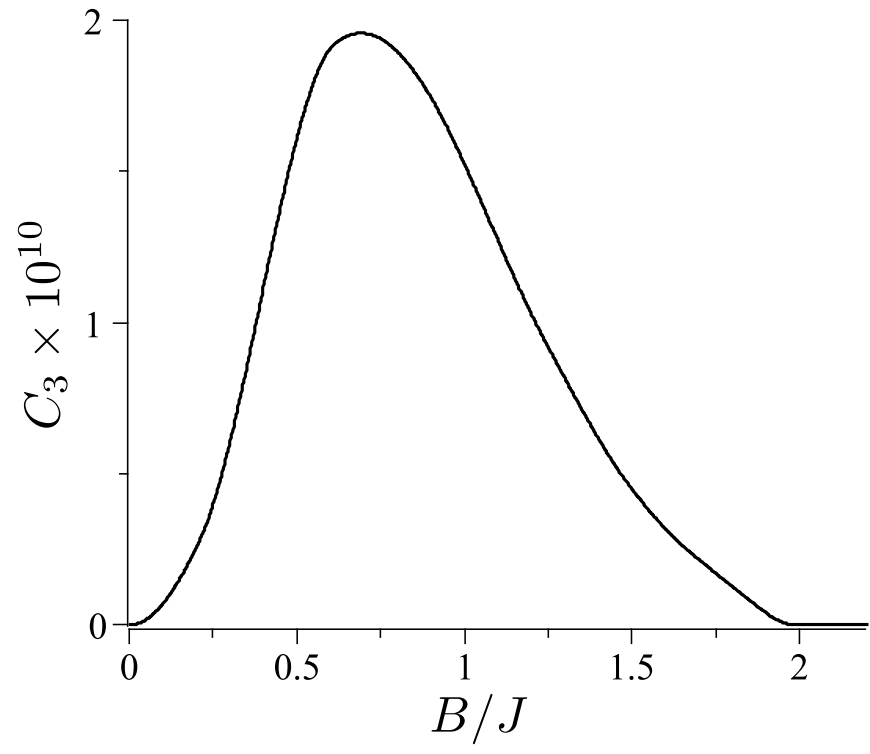

Figure 7. Contribution of the third level of the hierarchy of modes to $Z$ defined in Eq. (27); $L=40$. It is small compared with $C_{2}$ in Fig. 5 for the whole range of magnetic fields.

waves. These longitudinal waves propagate along the [010] direction that corresponds to the elastic mode $c_{22}$. Changes of the sound velocity $\delta v$ and the renormalised amplitude of the sound wave $1-Z$ were measured as functions of magnetic field at constant temperatures, using the experimental set up described in detail in Ref. 40 .

In Fig. 8 we compare the experimental data for the sound velocity with the theoretical results expressed in Eqs. (10[11). By fitting the static correlation function given by Eq. (10), we extract $J_{2} b^{2}=-24.5 J$, with the magnetic coupling constant $J=0.375 \mathrm{meV}$ taken from ${ }^{7}$ - see inset in Fig. 8. We find no signatures of the dynamical correlation functions represented by Eq. (11) which are parametrically small - down to the noise level of our experiment. This defines an upper bound to the other microscopic constant $J_{1} b \leq 1.25 \times 10^{4} \mathrm{~J}$.

Analysing the attenuation of the amplitude of the sound wave $Z$, we find that its functional dependence on the magnetic field is in good agreement with the dynamic hybridisation mechanism represented by Eq. (24) - see Fig. 8 and additional data in Fig. 9. By fitting its amplitude, we extract the other microscopic parameter as $J_{1} b=3563 \mathrm{~J} / \sqrt{I_{c}}$, where $I_{c}$ is the degree of nonmagnetic losses. A quantitative determination of these losses is not possible since they consist of various extrinsic (e.g. coupling and diffraction losses, non-parallel alignment of the sample, etc) and intrinsic attenuation mechanisms like direct scattering at defects or dislocation damping. $\underline{\underline{41}}$ However, even for $I_{c}=1$ this value of $J_{1} b$ is consistent with the bound from the measurement of the sound velocity.

The values of the microscopic constants are signifi- 


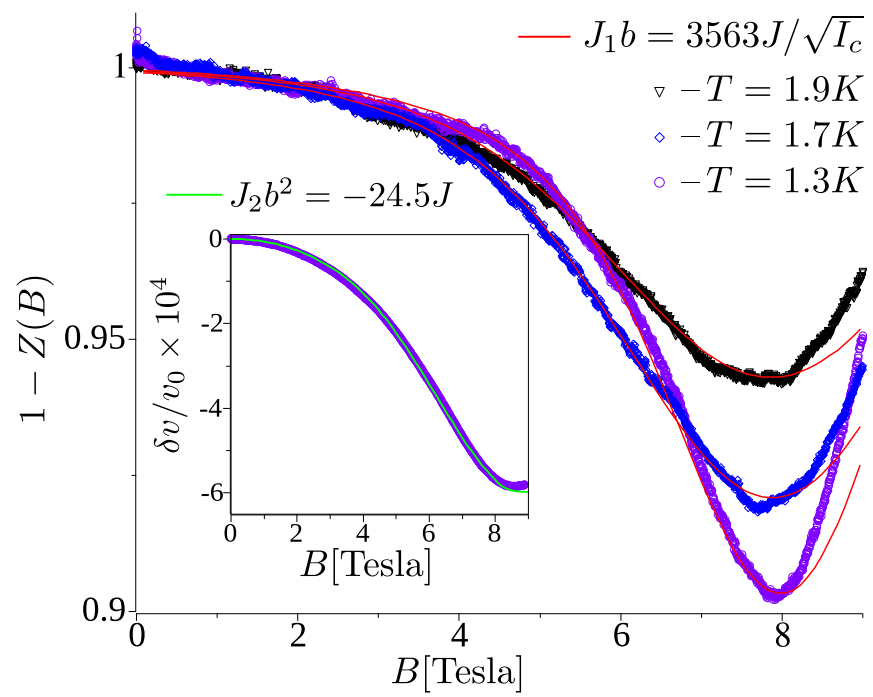

Figure 8. Experimental results (open triangles, diamonds, and circles) of the renormalised amplitude $1-Z$ of the longitudinal ultrasound wave propagating along the [010] axis of $\mathrm{Cs}_{2} \mathrm{CuCl}_{4}$ at $T=(1.900 \pm 0.005) \mathrm{K}, T=(1.700 \pm 0.005) \mathrm{K}$, and $T=(1.300 \pm 0.005) \mathrm{K}$. The red lines represent the results of Eq. (24) with $J_{1} b=3563 J / \sqrt{I_{c}}$. The inset shows data (open circles) of the corresponding normalised sound velocity at $T=(1.300 \pm 0.005) \mathrm{K}$. The green line shows the result of Eq. (10) using $J_{2} b^{2}=-24.5 J$. Additional data are presented in Fig. 9

cantly different from the values measured along the $a$ axis in Ref. 42 manifesting an anisotropy of $\mathrm{Cs}_{2} \mathrm{CuCl}_{4}$. Our very good fit of the magnetic field dependencies by the purely one-dimensional theory in Figs. 8 and 9 gives a further argument that the inter-chain interactions in $\mathrm{Cs}_{2} \mathrm{CuCl}_{4}$ in the finite temperature regime are negligible despite only a moderate degree of the exchange anisotropy of $\sim 3$ in the $a-b$ plane $\stackrel{43}{~}$

\section{CONCLUSIONS}

In conclusion, constructing a microscopic theory of magneto-elasticity in $1 \mathrm{D}$ we have shown that the necessary correlation functions involve the many-body excitations at all energy scales and have identified a new mechanism of sound attenuation. Our theoretical predictions agree with our ultrasound experiments in the $1 \mathrm{D}$ regime of $\mathrm{Cs}_{2} \mathrm{CuCl}_{4}$.

\section{ACKNOWLEDGEMENTS}

We acknowledge financial support by the DFG through the SFB/TRR 49.

\section{Appendix A: Derivation of the quantisation equation for pi-pairs}

The XXZ model is a generalisation of Heisenberg model that introduces the interaction strength between magnons $J \Delta$ as a model parameter, which breaks the rotational symmetry of the spin-spin interaction term $\mathbf{S}_{j} \cdot \mathbf{S}_{j+1}$. In one-dimension the model reads

$$
H_{m}=\sum_{j=1}^{L}\left(J \frac{S_{j}^{-} S_{j+1}^{+}+S_{j}^{+} S_{j+1}^{-}}{2}+J \Delta S_{j}^{z} S_{j+1}^{z}+B S_{j}^{z}\right),
$$

where $S_{j}^{ \pm}=S_{j}^{x} \pm i S_{j}^{y}$. For $\Delta=1$ this model becomes the model in Eq. (2). The $N$-magnon eigenstates of this Hamiltonian can be found by solving a set of Bethe equations,

$$
q_{j} L-\sum_{l \neq j} \varphi_{j l}=2 \pi I_{j}
$$

for $N$ quasimomenta $q_{j}$, where the two-magnon scattering phases are given by

$$
e^{i \varphi_{i j}}=-\frac{e^{i\left(q_{i}+q_{j}\right)}+1-2 \Delta e^{i q_{i}}}{e^{i\left(q_{i}+q_{j}\right)}+1-2 \Delta e^{i q_{j}}}
$$

and $I_{j}$ is a set of $N$ non-equal integer numbers.

In the free magnon limit $\Delta=0$ the two-body phase shifts $\varphi_{i j}$ become independent of quasimomenta and equal to the shifts of free fermions or hard-core bosons, $\varphi_{i j}=\pi$ that is immediately obtained by taking the $\Delta \rightarrow$ 0 limit of Eq. (A3) giving $\exp \left(i \varphi_{i j}\right)=-1$. This results restores the plain wave quantisation condition for each magnon independently, $q_{j}=2 \pi\left(I_{j}+1 / 2\right) / L, \stackrel{44}{\text { from the }}$ system of non-linear Bethe equations in Eq. (A2). Alternatively, the free magnon result can be obtained by setting $\Delta=0$ in the Hamiltonian in Eq. (A1) and then by diagonalising the resulting XY model directly $\underline{45}$

However, the non-interacting limit becomes ambiguous when at least one pair of quasimomenta in an $N$ magnon state satisfies the condition $q_{i}+q_{j}=2 \pi\left(I_{i}+I_{j}+1\right) / L=$ $\pm \pi$ at $\Delta=0$. In evaluating the $\Delta=0$ limit of Eq. (A3), the leading order of $e^{i\left(q_{i}+q_{j}\right)}+1$ is zero in the Taylor series around the $\Delta=0$ point, both in the numerator and in the denominator. Thus, higher order coefficients have to be calculated, $e^{i\left(q_{i}+q_{j}\right)}+1=c_{1} \Delta+c_{2} \Delta^{2}+\ldots$, that requires, in general, solving the whole set of $N$ Bethe equation in Eq. (A2) in a non-linear fashion, i.e. $c_{1}, c_{2}, \ldots$ depend on all $q_{l}$ - not just $q_{i}$ and $q_{j}$ - since $\Delta$ is still finite, which requires solving all $N$ Bethe equations for all $q_{l}$ simultaneously in taking the limit. This issue was noted in Refs. 46 and 47 but was never resolved. Here we start from a finite but small $\Delta$, for which all scattering phase are well-behaved, and then take the limit $\Delta \rightarrow 0$ systematically.

Let us consider a $N$-magnon solution of Bethe equations that containts $2 r$ quasimomenta that satisfy the 


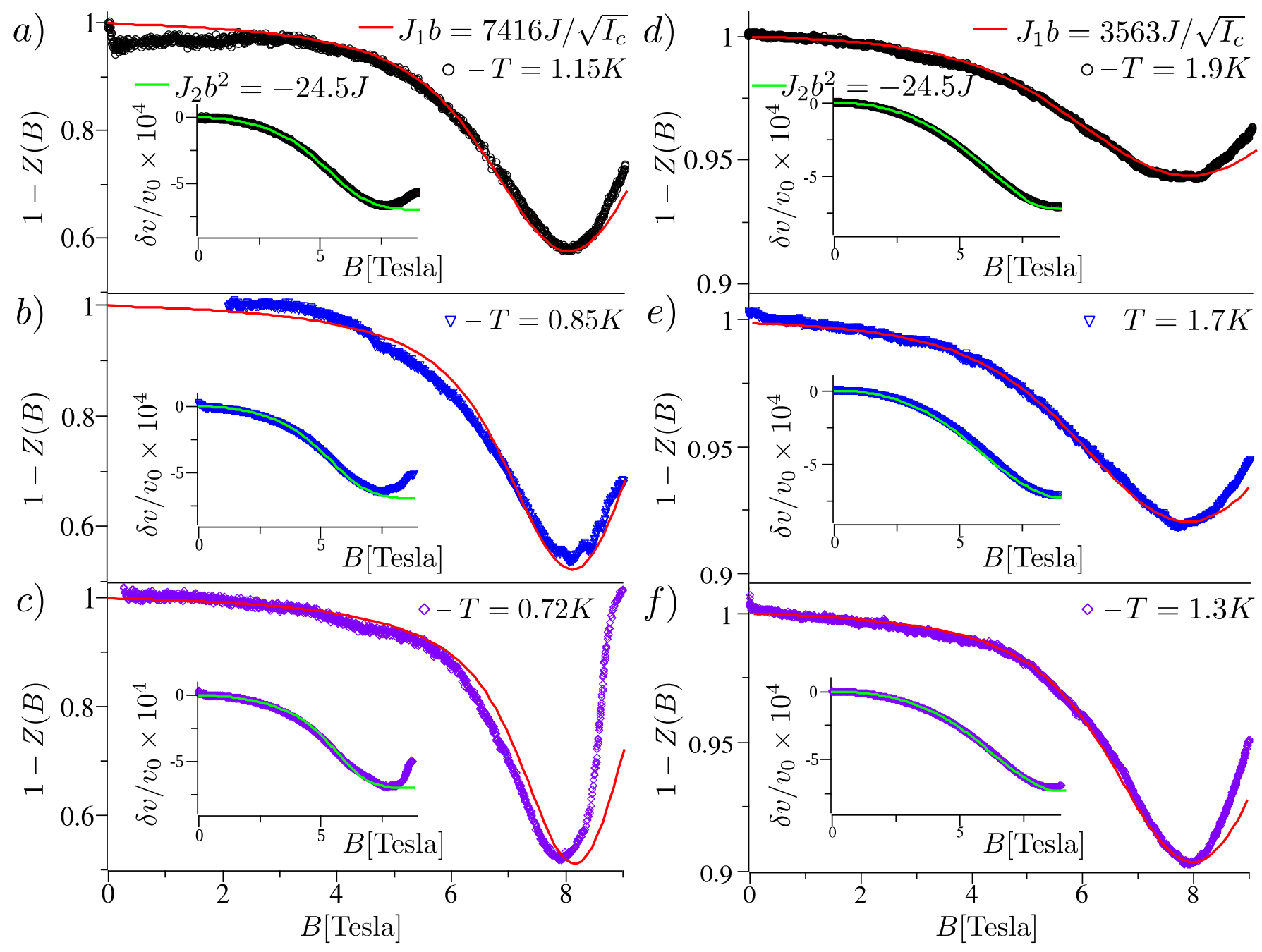

Figure 9. Experimental results (open circles, triangles, and diamonds) of the renormalised amplitude $1-Z$ of the longitudinal ultrasound wave propagating along [010] axis of $\mathrm{Cs}_{2} \mathrm{CuCl}_{4}$ at the temperatures of a) $\left.T=(1.150 \pm 0.005) \mathrm{K}, \mathrm{b}\right) T=(0.850 \pm 0.005)$ $\mathrm{K}$, c) $T=(0.720 \pm 0.005) \mathrm{K}, \mathrm{d}) T=(1.900 \pm 0.005) \mathrm{K}, \mathrm{e}) T=1.700 \pm 0.005) \mathrm{K}$, and f) $T=(1.300 \pm 0.005) \mathrm{K}$. The red lines represent the results of Eq. (24) with $J_{1} b=7416 J / \sqrt{I_{c}}$ for a,b,c) and with $J_{1} b=3563 / \sqrt{I_{c}}$ for d,e,f). The data in $\mathrm{d}, \mathrm{e}, \mathrm{f})$ were taken in a different cryostat system using upgraded electronics and a better quality sample compared with the data in $\mathrm{a}, \mathrm{b}, \mathrm{c})$ leading to a decrease of the non-magnetic losses by a factor of $I_{c}(a, b, c) / I_{c}(d, e, f) \approx 4.3$. The insets show data of the corresponding normalised sound velocities at the same temperatures. The green lines show the result of Eq. (10) using $J_{2} b^{2}=-24.5 J$. The results of our ultrasound experiments are still relatively close to the $1 \mathrm{D}$ regime at the temperature of $T=(0.720 \pm 0.005) \mathrm{K}$, at which the system is already in a transitional state between the $1 \mathrm{D}$ and a $2 \mathrm{D}$ regime.

$q_{2 j}+q_{2 j+1}= \pm \pi$ condition (we will call these pairs of quasimomenta pi-pairs below) and $N-2 r$ quasimomenta that do not have a pi-pair. For a finite but small $\Delta \ll 1$ the quasimomenta of a pi-pair can be parameterised as

$$
\begin{aligned}
q_{2 j} & = \pm \pi-\alpha_{j}+\frac{\delta_{j}}{2}, \quad j \leq r, \\
q_{2 j+1} & =\alpha_{j}+\frac{\delta_{j}}{2},
\end{aligned}
$$

where $\alpha_{j}$ is an unknown parameter of the $j^{\text {th }}$ pi-pair that has a non-zero value, since Bethe equations for this pair can not be solved due to the unknown (at the moment) phase shift $\varphi_{2 j, 2 j+1}$ at $\Delta=0$, and $\delta_{j}$ is a parameter that vanishes at $\Delta=0$. On the other hand, the remaining quasimomenta $j>2 r$ can be found immediately for $\Delta=$ 0 since all of their scattering phases in Eq. (A3) for these quasimomenta are well-behaved, $\varphi_{i j}=\pi$. Thus at a finite $\Delta \ll 1$ we can write

$$
q_{j}=\frac{2 \pi\left(I_{j}+\frac{1}{2}\right)}{L}+\epsilon_{j}, \quad j>2 r,
$$

where $\epsilon_{j}$ are small corrections due to a finite $\Delta$ that depend on all other quasimomenta and vanish for $\Delta=0$. Conservation of the total momentum of $N$ magnons, $\sum_{j=1}^{N} q_{j}=2 \pi \sum_{j=1}^{N} I_{j} / L$ including the pi-pairs, is independent of the interactions and imposes an additional 
constraint on $\delta_{j}$ and $\epsilon_{j}$,

$$
\sum_{j=2 r+1}^{N} \epsilon_{j}=-\sum_{j=1}^{r} \delta_{j}
$$

It is obtained as a sum of all equation in Eq. (A2) after substitution of Eqs. A4 A6).

Since $\alpha_{j}$ cannot be obtained directly from its own Bethe equation due to the undefined scattering phase within the corresponding pi-pair, we are going to obtain an equation for $\alpha_{j}$ from the other $j>2 r$ Bethe equations that do not have this issue. We start from expanding $\varphi_{j i}$ for $j>2 r$ magnons - which are defined at the point $\Delta=0-$ up to the linear order in small $\Delta$. Taking into account that $\epsilon_{j}$ is proportional to $\Delta$ and $\delta_{j}$ is linear (or a higher order) in $\Delta$ we obtain the following expansion of $\varphi_{j i}$ between the $j^{t h}$ magnon and a pi-pair and the $j^{t h}$ magnon and another $j^{\prime t h}$ magnons, which do not have a pi-pair, respectively,

$$
\begin{aligned}
\varphi_{j, 2 i} & =\pi-2 \Delta \frac{\sin \frac{q_{j}-\alpha_{i}}{2}}{\cos \frac{q_{j}+\alpha_{i}}{2}}, \\
\varphi_{j, 2 i+1} & =\pi-2 \Delta \frac{\cos \frac{q_{j}+\alpha_{i}}{2}}{\sin \frac{q_{j}-\alpha_{i}}{2}}, \\
\varphi_{j j^{\prime}} & =\pi-2 \Delta \frac{\sin \frac{q_{j}-q_{j^{\prime}}}{2}}{\cos \frac{q_{j}+q_{j^{\prime}}}{2}} .
\end{aligned}
$$

Then we substitute these expansions in Eq. A2 and sum all of them with $j>2 r$ obtaining a relation between $\delta_{i}$ and $\alpha_{i}$ that parameterise the quasimomenta for $j \leq 2 r$,

$$
-L \sum_{i=1}^{r} \delta_{i}+4 \Delta \sum_{i=1}^{r} \sum_{j=2 n+1}^{N} \frac{1-\sin q_{j} \sin \alpha_{i}}{\sin q_{j}-\sin \alpha_{i}}=0,
$$

where the sum over $j$ is taken over the remaining quasimomenta that do not have a pi-pair, given by Eq. (A6) with $\epsilon_{j}=0$. Here we used Eq. (A7) to express $\epsilon_{j}$ through $\delta_{j}$ and cancelled all $\varphi_{j j^{\prime}}$ terms for both $j, j^{\prime}>2 r$ due to the $\varphi_{j j^{\prime}}=-\varphi_{j^{\prime} j}$ symmetry of Eq. A10 - note that the scattering phases are defined up to an arbitrary period $2 \pi$ times an integer.

The equation in Eq. (A11) is a sum of $r$ terms and each term depends only on two unknown variables $\delta_{i}$ and $\alpha_{i}$. Thus Eq. (A11) splits into $r$ independent equations and solving them separately we find

$$
\delta_{i}=\frac{4}{L} \sum_{j=2 r+1}^{N} \frac{1-\sin q_{j} \sin \alpha_{i}}{\sin q_{j}-\sin \alpha_{i}} \Delta .
$$

This result shows that the linear term in the Taylor expansion for $\delta_{i}$ in $\Delta$ does not vanish. However it depends on the still unknown parameter $\alpha_{i}$. In order to find it, we take the $\Delta \rightarrow 0$ limit of Eq. (A3) for the two quasimomenta within the $i^{\text {th }}$ pi-pair and obtain

$$
e^{i \varphi_{2 i, 2 i+1}}=\frac{i \frac{2}{L} \sum_{j=2 r+1}^{N} \frac{1-\sin q_{j} \sin \alpha_{i}}{\sin q_{j}-\sin \alpha_{i}}-e^{-i \alpha_{i}}}{i \frac{2}{L} \sum_{j=2 r+1}^{N} \frac{1-\sin q_{j} \sin \alpha_{i}}{\sin q_{j}-\sin \alpha_{i}}+e^{i \alpha_{i}}},
$$

where

$$
e^{i\left(q_{2 i}+q_{2 i+1}\right)}+1=\frac{4}{L} \sum_{j=2 r+1}^{N} \frac{1-\sin q_{j} \sin \alpha_{i}}{\sin q_{j}-\sin \alpha_{i}} \Delta
$$

was expanded up to the linear order in $\Delta$, substituted in Eq. (A3), and $\Delta$ was cancelled from the whole expression altogether. Substituting Eq. (A13) into each of the $2 i^{t h}$ (or $2 i+1^{s t}$ ) Bethe equation in Eq. (A2) in the exponential form we obtain an equation for each $\alpha_{i}$ independently in the $\Delta=0$ limit,

$$
-e^{i L \alpha_{i}} \frac{i \frac{2}{L} \sum_{j=2 r+1}^{N} \frac{1-\sin q_{j} \sin \alpha_{i}}{\sin q_{j}-\sin \alpha_{i}}-e^{-i \alpha_{i}}}{i \frac{2}{L} \sum_{j=2 r+1}^{N} \frac{1-\sin q_{j} \sin \alpha_{i}}{\sin q_{j}-\sin \alpha_{i}}+e^{i \alpha_{i}}}=1 .
$$

This result does not coincide with the free wave quantisation condition $\exp \left(i \alpha_{i} L\right)=-1$, being a non-linear equation for $\alpha_{i}$. Its solutions can be found by introducing an extra deformation parameter $\lambda$,

$$
-e^{i L \alpha} \frac{i \frac{2 \lambda}{L} \sum_{j=2 r+1}^{N} \frac{1-\sin q_{j} \sin \alpha}{\sin q_{j}-\sin \alpha}-e^{-i \alpha}}{i \frac{2 \lambda}{L} \sum_{j=2 r+1}^{N} \frac{1-\sin q_{j} \sin \alpha}{\sin q_{j}-\sin \alpha}+e^{i \alpha}}=1,
$$

where the subscript was omitted, $\alpha_{i} \rightarrow \alpha$, since the equation is the same for all indices $i$. The solutions can be classified in the limit $\lambda=0$, like the Bethe equations, where Eq. (A16) is solved by $\alpha=$ $2 \pi\left(I_{j}+1 / 2\right) /(L-2)$. Then a smooth deformation of the equation from $\lambda=0$ to $\lambda=1$ gives all solutions of of the non-linear Eq. A15). The quantisation equation of pi-pairs in the $\Delta=0$ limit before Eq. (6) is Eq. (A16) in the logarithmic form.

The two-magnon solutions of Bethe equations that we identified as pi-pairs in Eq. (A16) at $\Delta=0$ can account for the missing complex solutions, which instead remain real, of the XXX model at $\Delta=1$ found in Ref. 48. For $N=2$ the parameter $\delta$ in Eq. (A12) remains zero for any $\Delta$ making the scattering phase $\varphi_{12}=-2 \alpha$ and Eq. (A2) independent of $\Delta$ as well, in this case. Thus this two-magnon solution remains real at $\Delta=1$ and has to be removed from the class of complex conjugated quasimomenta. We also note that pi-pairs are still solutions of Bethe equations at any finite $\Delta$ in full accord with the arguments of Ref. 49. It is only the limit $\Delta \rightarrow 0$ of these solutions that does not recover the single particle quantitation rule $q_{j}=2 \pi\left(I_{j}+1 / 2\right) / L$.

\section{Appendix B: Normalisation factors of Bethe states}

The eigenstates of the XXZ model in Eq. (A1) are the Bethe states

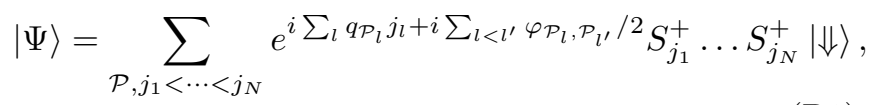


where $\mathcal{P}$ is a permutation of $N$ quasimomenta $q_{j}$ and $|\Downarrow\rangle$ is the ferromagnetic ground state. In this so-called coordinate representation the many-body states $|\Psi\rangle$ are not factorisable making calculations of scalar products and expectation values in this representation almost intractable. However, a calculation of the form factors needed in Eq. (13) becomes manageable using the algebraic form of Bethe ansatz $\stackrel{32}{\underline{2}}$ in which Bethe states are factorised in terms of operators with given commutation relations.

Following Ref. 32 we write down the many-body wave functions using operators that satisfy a Yang-Baxter algebra as

$$
|\mathbf{u}\rangle=\prod_{j=1}^{N} C\left(u_{j}\right)|\Downarrow\rangle,
$$

where $u_{j}$ are $N$ auxiliary parameters and $C(u)$ is one of the four matrix elements of the transition matrix

$$
T(u)=\left(\begin{array}{ll}
A(u) & B(u) \\
C(u) & D(u)
\end{array}\right),
$$

which is defined in an auxiliary $2 \times 2$ space. This $T$-matrix satisfies the Yang-Baxter equation

$$
R(u-v)(T(u) \otimes T(v))=(T(v) \otimes T(u)) R(u-v) .
$$

Here we use the following $R$-matrix that corresponds to the spin Hamiltonian in Eq.(A1),

$$
\begin{aligned}
& R(u)=\left(\begin{array}{llll}
1 & & & \\
& b(u) & c(u) & \\
& c(u) & b(u) & \\
& & & 1
\end{array}\right) \\
& A_{u} \prod_{j=1}^{N} C\left(u_{j}\right)|0\rangle=a_{u} \prod_{j=1}^{N} \frac{1}{b_{u j}} C\left(u_{j}\right)|0\rangle-\sum_{j=1}^{N} a_{j} \frac{c_{u j}}{b_{u j}} C(u) \prod_{l=1 \neq j}^{N} \frac{1}{b_{j l}} C\left(u_{l}\right)|\Downarrow\rangle, \\
& D_{u} \prod_{j=1}^{N} C\left(u_{j}\right)|0\rangle=d_{u} \prod_{j=1}^{N} \frac{1}{b_{j u}} C\left(u_{j}\right)|0\rangle+\sum_{j=1}^{N} d_{j} \frac{c_{u j}}{b_{u j}} C(u) \prod_{l=1 \neq j}^{N} \frac{1}{b_{l j}} C\left(u_{l}\right)|\Downarrow\rangle,
\end{aligned}
$$

where $b(u)=\sinh (u) / \sinh (u+2 \eta)$ and $c(u)=$ $\sinh (2 \eta) / \sinh (u+2 \eta)$.

The entries of Eq. (B4) give commutation relations between the matrix elements of $T$. Here we write down four of them that will be used later,

$$
\begin{gathered}
{\left[B_{u}, C_{v}\right]=\frac{c(u-v)}{b(u-v)}\left(A_{u} D_{v}-A_{v} D_{u}\right),} \\
A_{u} C_{v}=\frac{1}{b(u-v)} C_{v} A_{u}-\frac{c(u-v)}{b(u-v)} C_{u} A_{v}, \\
D_{u} C_{v}=\frac{1}{b(v-u)} C_{v} D_{u}-\frac{c(v-u)}{b(v-u)} C_{u} D_{v}, \\
{\left[A_{u}, D_{v}\right]=\frac{c(u-v)}{b(u-v)}\left(C_{v} B_{u}-C_{u} B_{v}\right) .}
\end{gathered}
$$

We have introduced the subscript $u$ and $v$ as a shorthand of the argument, e.g. $A_{u} \equiv A(u)$, above.

The transfer matrix $\tau(u)=\operatorname{Tr} T(u)=A(u)+D(u)$ contains all of the conserved quantities of the model in Eq. (A1) including the Hamiltonian. Thus if $|\mathbf{u}\rangle$ is a eigenstate of $\tau(u)$ then it is an eigenstate of the Hamiltonian. The eigenvalue equation, $\tau(u)|\mathbf{u}\rangle=\mathcal{T}_{u}|\mathbf{u}\rangle$ where $\mathcal{T}_{u}$ is a scalar quantity - the corresponding eigenvalue, can be solved using the commutation relations in Eqs. (B6, B9). The results of acting with the $A_{u}$ and $D_{u}$ operators on the state $|\mathbf{u}\rangle$ in Eq. (B2) are obtained by commuting them from left to right through the product where the vacuum eigenvalues of the operators, $A_{u}|\Downarrow\rangle=$ $a_{u}|\Downarrow\rangle$ and $D_{u}|\Downarrow\rangle=d_{u}|\Downarrow\rangle$, are

$$
a_{u}=\frac{\cosh ^{L}(u-\eta)}{\cosh ^{L}(u+\eta)} \quad \text { and } \quad d_{u}=1 .
$$

Since the right hand side of Eqs. (B10|B11 contains terms that are not proportional to the original state multiplied by a scalar, an arbitrary Bethe state is not an eigenstate of the transfer matrix $\tau$ for an arbitrary set of the auxiliary parameters $u_{j}$. However, the second terms 
from Eq. (B5) gives the following Bethe equation and the eigenvalue of the transfer matrix $\tau$,

$$
\frac{\cosh \left(u_{j}-\eta\right)^{L}}{\cosh \left(u_{j}+\eta\right)^{L}}=\prod_{l=1 \neq j}^{N} \frac{\sinh \left(u_{j}-u_{l}-2 \eta\right)}{\sinh \left(u_{j}-u_{l}+2 \eta\right)}
$$

$$
\mathcal{T}_{u}=a_{u} \prod_{j=1}^{N} \frac{1}{b_{u j}}+d_{u} \prod_{j=1}^{N} \frac{1}{b_{j u}}
$$

The Bethe ansatz equations - in the coordinate representation - are obtained under substitution of

$$
u_{j}=\ln \left[\sqrt{\frac{1-e^{i q_{j}-2 \eta}}{1-e^{-i q_{j}-2 \eta}}}\right]-\frac{i q_{j}}{2}
$$

and

$$
\eta=\frac{\operatorname{acosh} \Delta}{2}
$$

into Eq. B14.

The scalar product between two Bethe states $\langle\mathbf{v}|$ and $|\mathbf{u}\rangle$ can be calculated using the commutation relations in Eqs. (B6, B9 . The multiplication of the bra and ket states in the form of Eq. (B2) is evaluated by commuting each operator $B\left(v_{j}\right)$ from left to right through the product of $C\left(u_{j}\right)$ operators and then by using the vacuum eigenvalues of the generated $A$ and $D$ operators from Eq. (B12). When $u_{j}$ is a solution of Eq. (B14) and $v_{j}$ is an arbitrary set of auxiliary parameters the result can be written in a compact form as a determinant of an $N \times N$ matrix - the so-called Slavnov's formula, 50

$$
\langle\mathbf{v} \mid \mathbf{u}\rangle=\frac{\prod_{i, j=1}^{N} \sinh \left(v_{j}-u_{i}\right)}{\prod_{j<i} \sinh \left(v_{j}-v_{i}\right) \prod_{j<i} \sinh \left(u_{j}-u_{i}\right)} \operatorname{det} \hat{T},
$$

where matrix elements are $T_{a b}=\partial_{u_{a}} \mathcal{T}\left(v_{b}\right)$. Under substitution of $\mathcal{T}(u)$ from Eq. (B5) these matrix elements read

$$
T_{a b}=\frac{\cosh ^{L}\left(v_{b}-\eta\right)}{\cosh ^{L}\left(v_{b}+\eta\right)} \frac{\sinh (2 \eta)}{\sinh ^{2}\left(v_{b}-u_{a}\right)} \prod_{j=1 \neq a}^{N} \frac{\sinh \left(v_{b}-u_{j}+2 \eta\right)}{\sinh \left(v_{b}-u_{j}\right)}-\frac{\sinh (2 \eta)}{\sinh ^{2}\left(u_{a}-v_{b}\right)} \prod_{j=1 \neq a}^{N} \frac{\sinh \left(u_{j}-v_{b}+2 \eta\right)}{\sinh \left(u_{j}-v_{b}\right)} .
$$

The normalisation factor of Bethe states in Eq. (B2) can be evaluated by taking the $\mathbf{v} \rightarrow \mathbf{u}$ limit of Eq. (B18)

$$
\langle\mathbf{u} \mid \mathbf{u}\rangle=\sinh ^{N}(2 \eta) \prod_{i \neq j=1}^{N} \frac{\sinh \left(u_{j}-u_{i}+2 \eta\right)}{\sinh \left(u_{j}-u_{i}\right)} \operatorname{det} \hat{M}
$$

where the matrix elements are

$$
M_{a b}= \begin{cases}-L \frac{\sinh 2 \eta}{\cosh \left(u_{a}+\eta\right) \cosh \left(u_{a}-\eta\right)}-\sum_{j \neq a} \frac{\sinh 4 \eta}{\sinh 4 \eta} \frac{u_{a}\left(u_{a}-u_{j}-2 \eta\right) \sinh \left(u_{a}-u_{j}+2 \eta\right)}{\sinh } & , a=b \\ \frac{\sinh \left(u_{b}-u_{a}+2 \eta\right) \sinh \left(u_{b}-u_{a}-2 \eta\right)}{} & , a \neq b\end{cases}
$$

\section{Appendix C: Derivation of the dynamical matrix element for spins}

In this section we will calculate the matrix element $\left\langle\mathbf{q}\left|\mathbf{S}_{1} \cdot \mathbf{S}_{2}\right| 0\right\rangle$ - with respect to Bethe states of the spin Hamiltonian - that is needed for evaluating Eq. (11). We start by splitting the matrix element of the scalar product $\mathbf{S}_{1} \cdot \mathbf{S}_{2}$ into three parts,

$$
\left\langle\mathbf{q}\left|\mathbf{S}_{1} \cdot \mathbf{S}_{2}\right| 0\right\rangle_{m}=G_{+-}+G_{-+}+G_{z z},
$$

where

$$
G_{+-}=\frac{1}{2}\left\langle\mathbf{v}\left|S_{1}^{+} S_{2}^{-}\right| \mathbf{u}\right\rangle
$$

$$
G_{-+}=\frac{1}{2}\left\langle\mathbf{v}\left|S_{1}^{-} S_{2}^{+}\right| \mathbf{u}\right\rangle
$$

$$
G_{z z}=\left\langle\mathbf{v}\left|S_{1}^{z} S_{2}^{z}\right| \mathbf{u}\right\rangle,
$$

$u_{j}$ are the quasimomenta of the ground state $|0\rangle$, and $v_{j}$ are the quasimomenta of an excited state $|\mathbf{q}\rangle$ with the same number of particles.

The local spin operators of the model in Eq. (A1) can be expressed in terms of the algebraic Bethe ansatz 
operators from Eq. (B3) as $16,17,51$

$$
\begin{aligned}
& S_{1}^{+}=C_{\xi} \tau_{\xi}^{L-1}, S_{2}^{+}=\tau_{\xi} C_{\xi} \tau_{\xi}^{L-2}, \\
& S_{1}^{-}=B_{\xi} \tau_{\xi}^{L-1}, S_{2}^{-}=\tau_{\xi} B_{\xi} \tau_{\xi}^{L-2}, \\
& S_{1}^{z}=S_{2}^{z} \frac{A_{\xi}-D_{\xi}}{2} \tau_{\xi}^{L-1}, S_{2}^{z}=\tau_{\xi} \frac{A_{\xi}-D_{\xi}}{2} \tau_{\xi}^{L-2},
\end{aligned}
$$

where $\xi=-i \pi / 2+\eta$.

Firstly, we evaluate the +- correlation function. Un- der the substitution of Eqs. (C5, C6) in to Eq. (C2) it reads

$$
G_{+-}=\frac{1}{2}\left\langle\mathbf{v}\left|C_{\xi} B_{\xi}\right| \mathbf{u}\right\rangle
$$

Commuting of the $B_{\xi}$ operator from left to right through a product of $C\left(u_{j}\right)$ operators by means of the commutation relations in Eqs. (B6, B9 gives

$$
B_{\xi} \prod_{j=1}^{N} C_{u_{j}}|\Downarrow\rangle=\sum_{x=1}^{N+1} a_{x} c_{x \xi} \prod_{i=1 \neq x}^{N+1} \frac{1}{b_{x i}} \sum_{y=1 \neq x}^{N+1} c_{\xi y} \prod_{j=1 \neq x, y}^{N+1} \frac{1}{b_{j y}} \prod_{j=1 \neq x, y}^{N+1} C_{u_{j}}|\Downarrow\rangle
$$

where $u_{N+1} \equiv \xi$. Multiplying the above expression by $C_{\xi}$ and evaluating the scalar product with the final state $\langle\mathbf{v}|$ we obtain

$$
\begin{aligned}
G_{+-}= & \frac{1}{2} \sum_{x=1}^{N} a_{x} \frac{c_{x \xi}}{b_{x \xi}} \prod_{i=1 \neq x}^{N} \frac{1}{b_{x i}} \sum_{y=1 \neq x}^{N} \frac{c_{\xi y}}{b_{\xi y}} \prod_{j=1 \neq x, y}^{N} \frac{1}{b_{j y}}\left\langle u_{x-1}, \xi, u_{x+1}, u_{y-1}, \xi, u_{y+1} \mid \mathbf{v}\right\rangle \\
& +\frac{1}{2} \sum_{x=1}^{N} a_{x} \frac{c_{x \xi}}{b_{x \xi}} \prod_{i=1 \neq x}^{N} \frac{1}{b_{x i}} \prod_{j=1 \neq x}^{N} \frac{1}{b_{j \xi}}\left\langle u_{x-1}, \xi, u_{x+1} \mid \mathbf{v}\right\rangle .
\end{aligned}
$$

Here the property $\langle\mathbf{v} \mid \mathbf{u}\rangle=\langle\mathbf{u} \mid \mathbf{v}\rangle$ where $v_{j}$ satisfy the Bethe equations and $u_{j}$ is an arbitrary set of auxiliary parameters 16,17 was used.

The remaining scalar product can be evaluated us- ing the Slavnov's formula (B18). By substituting $\xi=$ $-i \pi / 2+\eta$ into $\left\langle u_{x-1}, \xi, u_{x+1} \mid \mathbf{v}\right\rangle$ in the second line of Eq. (C10) explicitly we obtain

$$
\left\langle u_{x+1}, \xi, u_{x-1} \mid \mathbf{v}\right\rangle=\frac{i(-1)^{x} \prod_{j}^{N} \cosh \left(v_{j}+\eta\right) \prod_{j, i \neq x}^{N} \sinh \left(u_{i}-v_{j}\right) \operatorname{det} \hat{T}^{(x)}}{\prod_{j \neq x}^{N} \cosh \left(u_{j}-\eta\right) \prod_{i<j} \sinh \left(v_{i}-v_{j}\right) \prod_{i<j \neq x} \sinh \left(u_{i}-u_{j}\right)}
$$

where the matrix elements are

$$
\begin{gathered}
T_{a b}^{(x)}=T_{a b}, \quad b \neq x, \\
T_{a x}^{(x)}=\frac{\sinh (2 \eta)}{\cosh \left(v_{a}-\eta\right) \cosh \left(v_{a}+\eta\right)}, \quad b=x,
\end{gathered}
$$

and $T_{a b}$ are given in Eq. (B19).

Substitution of the two identical $u_{j}=u_{j^{\prime}}=\xi$ into the scalar product $\left\langle u_{x-1}, \xi, u_{x+1}, u_{y-1}, \xi, u_{y+1} \mid \mathbf{v}\right\rangle$ in the first line in Eq. (C10) makes the prefactor in Eq. (B18) divergent, i.e. the prefactor has a pole of the first order as a function of $\left(u_{j^{\prime}}-u_{j}\right)$. Simultaneously, the determinant in Eq. (B18) becomes zero under the same substitution $u_{j}=u_{j^{\prime}}=\xi$ since two lines of the matrix in Eq. (B19) becomes identical. Thus, we will derive the explicit expression for the whole scalar product by substituting $u_{j}=\xi$ first, then, by taking the limit $u_{j^{\prime}}=\bar{\xi} \rightarrow \xi$. Expanding the matrix elements in Eq. (B19) in a Taylor series in $(\bar{\xi}-\xi)$ and using general matrix identities we obtain

$$
\left|\begin{array}{c}
\ldots \\
\mathbf{A}^{T} \\
\cdots \\
\mathbf{A}^{T}+\left(\beta \mathbf{A}^{T}+\mathbf{X}^{T}\right)(\bar{\xi}-\xi) \\
\ldots
\end{array}\right|=(\bar{\xi}-\xi)\left|\begin{array}{c}
\cdots \\
\mathbf{A}^{T} \\
\cdots \\
\mathbf{X}^{T} \\
\cdots
\end{array}\right|,
$$

where

$$
A_{a}=\frac{\sinh (2 \eta)}{\cosh \left(v_{a}-\eta\right) \cosh \left(v_{a}+\eta\right)} \prod_{j}^{N} \frac{\cosh \left(v_{j}+\eta\right)}{\cosh \left(v_{j}-\eta\right)}
$$

is the $j^{\text {th }}$ row of Eq. (B19) under the substitution $u_{j}=\xi$,

$$
X_{a}=\frac{\sinh 2 \eta \sinh 2 v_{a}}{\cosh ^{2}\left(v_{a}-\eta\right) \cosh ^{2}\left(v_{a}+\eta\right)} \prod_{j}^{N} \frac{\cosh \left(v_{j}+\eta\right)}{\cosh \left(v_{j}-\eta\right)}
$$


is the linear coefficient in the Taylor expansion of the $j^{\text {th }}$ row of Eq. (B19) around the point $u_{j^{\prime}}=\xi$, which is not collinear with $A_{a}$ in the vector space, and $\beta$ is the part of the linear coefficient that is collinear with $A_{a}$.

Cancellation of the $\bar{\xi}-\xi$ from the denominator in
Eq. (C14) with the $1 /(\bar{\xi}-\xi)$ from the prefactor in Eq. (B18) makes the whole scalar product finite. Contributions of the orders higher than one (in the expansion of the determinant) vanish in the limit $\bar{\xi} \rightarrow \xi$ and we obtain

$$
\left\langle u_{x-1}, \xi, u_{x+1}, u_{y-1}, \xi, u_{y+1} \mid \mathbf{v}\right\rangle=(-1)^{x+y} \frac{\prod_{j} \cosh ^{2}\left(v_{j}+\eta\right)}{\prod_{j \neq x, y} \cosh ^{2}\left(u_{j}-\eta\right)} \frac{\prod_{j, j^{\prime} ; j^{\prime} \neq x, y} \sinh \left(u_{j^{\prime}}-v_{j}\right) \operatorname{det} \hat{T}^{(x y)}}{\prod_{j<j^{\prime}} \sinh \left(v_{i}-v_{j}\right) \prod_{j<j^{\prime} \neq x, y} \sinh \left(u_{i}-u_{j}\right)},
$$

where the matrix elements are

$$
T_{a b}^{(x y)}= \begin{cases}T_{a b} & , b \neq x, y \\ T_{a b}^{(b)} & , b=\min (x, y) \\ \frac{\sinh 2 \eta \sinh 2 v_{a}}{\cosh ^{2}\left(v_{a}-\eta\right) \cosh ^{2}\left(v_{a}+\eta\right)} & , b=\max (x, y)\end{cases}
$$

Secondly, we turn to evaluating the -+ correlation function. Under the substitution of Eqs. (C5, C6) into Eq. (C3) it reads

$$
G_{-+}=\frac{1}{2}\left\langle\mathbf{v}\left|B_{\xi} C_{\xi}\right| \mathbf{u}\right\rangle .
$$

When $B_{\xi}$ is commuted through the product of $C_{u_{j}}$ operators using the general result in Eq. (C9), the first step of commuting $B_{\xi}$ with $C_{\xi}$ introduces a divergent denominator through the commutation relation in Eq. (B6). However, the operator factor in the numerator of Eq. (B6) becomes zero at the same time making the whole expression finite. Since the divergence occurs at the first step of commuting $B_{\xi}$ through a product of $N+1$ operators $C\left(u_{j}\right)$, taking the limit after using Eq. (C9), as it is done in Ref. 17, creates an extra and significant computation problems: the original divergence spreads through many terms under the sum in Eq. (C9) and cancelling them explicitly is a complicated problem.

Here we will do it in a different way by cancelling this intermediate divergence from the beginning in Eq. (C19). Expanding the numerator and the denominator of the commutation relation in Eq. (B6) up to the linear order in $\bar{\xi}-\xi$, where $u \rightarrow \bar{\xi}$ and $v \rightarrow \xi$ auxiliary parameters were relabeled, we cancel the $\bar{\xi}-\xi$ with $1 /(\bar{\xi}-\xi)$. Then, substituting the result of this procedure in Eq. (C19) we obtain

$$
\begin{aligned}
G_{-+}= & \frac{1}{2}\left\langle\mathbf{v}\left|C_{\xi} B_{\xi}\right| \mathbf{u}\right\rangle+\frac{\sinh 2 \eta}{2} \\
& \times \lim _{\bar{\xi} \rightarrow \xi} \partial_{\bar{\xi}}\left(\left\langle\mathbf{v}\left|A_{\bar{\xi}} D_{\xi}\right| \mathbf{u}\right\rangle-\left\langle\mathbf{v}\left|A_{\xi} D_{\bar{\xi}}\right| \mathbf{u}\right\rangle\right),
\end{aligned}
$$

where $\left\langle\mathbf{v}\left|C_{\xi} B_{\xi}\right| \mathbf{u}\right\rangle$ has already been calculated in Eq. (C10).

The remaining two correlation functions under the derivative in Eq. (C20) can be calculated by successive use of the general result of commuting $A_{u}$ and $D_{v}$ operators through a product of $C\left(u_{j}\right)$ operators in Eqs. (B10, B11). The scalar product of $\langle\mathbf{v}|$ with the result of the commutation procedure gives

$$
\begin{aligned}
\left\langle\mathbf{v}\left|A_{\bar{\xi}} D_{\xi}\right| \mathbf{u}\right\rangle= & a_{\bar{\xi}} \prod_{l=1}^{N} \frac{1}{b_{l \xi}} \prod_{j=1}^{N} \frac{1}{b_{\bar{\xi} j}} \delta_{\mathbf{u}, \mathbf{v}}-\prod_{l=1}^{N} \frac{1}{b_{l \xi}} \sum_{j=1}^{N} a_{j} \frac{c_{\bar{\xi} j}}{b_{\bar{\xi} j}} \prod_{l=1 \neq j}^{N} \frac{1}{b_{j l}}\left\langle u_{j-1}, \bar{\xi}, u_{j+1} \mid \mathbf{v}\right\rangle \\
& +\sum_{j=1}^{N} \frac{c_{\xi j}}{b_{\xi j}} \prod_{l=1 \neq j}^{N} \frac{1}{b_{l j}} a_{\bar{\xi}} \frac{1}{b_{\bar{\xi} \xi}} \prod_{l=1 \neq j}^{N} \frac{1}{b_{\bar{\xi} l}}\left\langle u_{j-1}, \xi, u_{j+1} \mid \mathbf{v}\right\rangle \\
& -\sum_{j=1}^{N} \frac{c_{\xi j}}{b_{\xi j}} \prod_{l=1 \neq j}^{N} \frac{1}{b_{l j}} \sum_{j^{\prime}=1 \neq j}^{N} a_{j^{\prime}} \frac{c_{\bar{\xi} j^{\prime}}}{b_{\bar{\xi} j^{\prime}}} \frac{1}{b_{j^{\prime} \xi}} \prod_{l=1 \neq j, j^{\prime}}^{N} \frac{1}{b_{j^{\prime} l}}\left\langle u_{j-1}, \xi, u_{j+1}, u_{j^{\prime}-1}, \bar{\xi} u_{j^{\prime}+1} \mid \mathbf{v}\right\rangle \\
& -\sum_{j=1}^{N} \frac{c_{\xi j}}{b_{\xi j}} \prod_{l=1 \neq j}^{N} \frac{1}{b_{l j}} a_{\xi} \frac{c_{\bar{\xi} \xi}}{b_{\bar{\xi} \xi}} \prod_{l=1 \neq j}^{N} \frac{1}{b_{\xi l}}\left\langle u_{j-1}, \bar{\xi}, u_{j+1} \mid \mathbf{v}\right\rangle,
\end{aligned}
$$




$$
\begin{aligned}
\left\langle\mathbf{v}\left|A_{\xi} D_{\bar{\xi}}\right| \mathbf{u}\right\rangle= & a_{\bar{\xi}} \prod_{l=1}^{N} \frac{1}{b_{l \xi}} \prod_{j=1}^{N} \frac{1}{b_{\bar{\xi} j}} \delta_{\mathbf{u}, \mathbf{v}}-\prod_{l=1}^{N} \frac{1}{b_{l \xi}} \sum_{j=1}^{N} a_{j} \frac{c_{\bar{\xi} j}}{b_{\bar{\xi} j}} \prod_{l=1 \neq j}^{N} \frac{1}{b_{j l}}\left\langle u_{j-1}, \bar{\xi}, u_{j+1} \mid \mathbf{v}\right\rangle \\
& +\sum_{j=1}^{N} \frac{c_{\xi j}}{b_{\xi j}} \prod_{l=1 \neq j}^{N} \frac{1}{b_{l j}} a_{\bar{\xi}} \frac{1}{b_{\bar{\xi} \xi}} \prod_{l=1 \neq j}^{N} \frac{1}{b_{\bar{\xi} l}}\left\langle u_{j-1}, \xi, u_{j+1} \mid \mathbf{v}\right\rangle \\
& -\sum_{j=1}^{N} \frac{c_{\xi j}}{b_{\xi j}} \prod_{l=1 \neq j}^{N} \frac{1}{b_{l j}} \sum_{j^{\prime}=1 \neq j}^{N} a_{j^{\prime}} \frac{c_{\bar{\xi} j^{\prime}}}{b_{\bar{\xi} j^{\prime}}} \frac{1}{b_{j^{\prime}}} \prod_{l=1 \neq j, j^{\prime}}^{N} \frac{1}{b_{j^{\prime} l}}\left\langle u_{j-1}, \xi, u_{j+1}, u_{j^{\prime}-1}, \bar{\xi} u_{j^{\prime}+1} \mid \mathbf{v}\right\rangle
\end{aligned}
$$

for the both terms in the second line of Eq. (C20) respectively. Then, after taking the derivative of Eqs. (C21 C22), with respect to $\bar{\xi}$ and the limit $\bar{\xi} \rightarrow \xi$, we substitute the results in to Eq. (C20) and obtain

$$
\begin{aligned}
G_{-+} & =G_{+-}+\frac{\sinh 2 \eta}{2}\left[\prod_{l=1}^{N} \frac{1}{b_{l \xi}} \sum_{j=1}^{N} \sum_{l=1}^{N}\left[\tanh \left(u_{l}+\eta\right)-\tanh \left(v_{l}-\eta\right)\right] a_{j} \frac{c_{j \xi}}{b_{j \xi}} \prod_{l=1 \neq j}^{N} \frac{1}{b_{j l}}\left\langle u_{j-1}, \xi, u_{j+1} \mid \mathbf{v}\right\rangle\right. \\
& +\sum_{j=1}^{N} \frac{c_{j \xi}}{b_{j \xi}} \prod_{l=1 \neq j}^{N} \frac{1}{b_{l j}} \sum_{j^{\prime}=1 \neq j}^{N} a_{j^{\prime}} \frac{c_{\xi j^{\prime}}}{b_{\xi j^{\prime}}}\left(\tanh \left(u_{j^{\prime}}+\eta\right)-\tanh \left(u_{j}-\eta\right)\right) \frac{1}{b_{j^{\prime} \xi}} \prod_{l=1 \neq j, j^{\prime}}^{N} \frac{1}{b_{j^{\prime} l}}\left\langle u_{j-1}, \xi, u_{j+1}, u_{j^{\prime}-1}, \xi u_{j^{\prime}+1} \mid \mathbf{v}\right\rangle \\
& \left.+\prod_{l=1}^{N} \frac{1}{b_{l \xi}} \sum_{j=1}^{N} a_{j} \frac{c_{j \xi}}{b_{j \xi}} \prod_{l=1 \neq j}^{N} \frac{1}{b_{j l}}\left\langle u_{j-1}, \xi, u_{j+1} \mid \mathbf{v}\right\rangle^{\prime}\right],
\end{aligned}
$$

where the derivative of $\left\langle u_{j-1}, \bar{\xi}, u_{j+1} \mid \mathbf{v}\right\rangle$ with respect to $\bar{\xi}$ results in an additional determinant,

$$
\left\langle u_{x-1}, \xi, u_{x+1} \mid \mathbf{v}\right\rangle^{\prime}=\frac{i(-1)^{j} \prod_{j^{\prime}}^{N} \cosh \left(v_{j^{\prime}}+\eta\right) \prod_{j^{\prime}, i \neq x}^{N} \sinh \left(u_{i}-v_{j^{\prime}}\right) \operatorname{det} \hat{T}^{\prime}(x)}{\prod_{j^{\prime} \neq x}^{N} \cosh \left(u_{j^{\prime}}-\eta\right) \prod_{i<j^{\prime}} \sinh \left(v_{i}-v_{j^{\prime}}\right) \prod_{i<j^{\prime} \neq x} \sinh \left(u_{i}-u_{j^{\prime}}\right)},
$$

where the matrix elements are

$$
T_{a x}^{\prime(x)}=\frac{2 \sinh 2 \eta \tanh \left(v_{a}-\eta\right)}{\cosh \left(v_{a}-\eta\right) \cosh \left(v_{a}+\eta\right)}-\frac{\sinh ^{2} 2 \eta}{\cosh \left(v_{a}-\eta\right) \cosh \left(v_{a}+\eta\right)} \sum_{j=1 \neq a}^{N} \frac{1}{\cosh \left(v_{j}-\eta\right) \cosh \left(v_{j}+\eta\right)}
$$

for $b=x$ and the remaining entries for $b \neq x$ are $T_{a b}^{\prime}(x) \equiv T_{a b}$ from Eq. (B19).

Thirdly, we evaluate the $z z$ correlation function. Under the substitution of Eq. (C7) in Eq. (C4) it reads

$$
G_{z z}=\frac{1}{4}\left\langle\mathbf{v}\left|\left(A_{\xi}-D_{\xi}\right)\left(A_{\xi}-D_{\xi}\right)\right| \mathbf{u}\right\rangle
$$

Before proceeding with the commutation procedure as in the two previous cases we rewrite the above expression in a form more convenient for such a calculation using the definition of the transfer matrix, $A_{\xi}-D_{\xi}=2 A_{\xi}-\tau_{\xi}$, and its eigenvalue $\tau_{\xi}|\mathbf{u}\rangle=\mathcal{T}_{\xi}|\mathbf{u}\rangle$,

$$
G_{z z}=\frac{1}{2}\left\langle\mathbf{v}\left|A_{\xi}^{2}-\mathcal{T}_{\xi} A_{\xi}-D_{\xi} A_{\xi}\right| \mathbf{u}\right\rangle
$$

where $\mathcal{T}_{\xi}=\prod_{j} b_{j \xi}^{-1}$ is given by Eq. (B15) and we have assumed that $\langle\mathbf{v}|$ and $|\mathbf{u}\rangle$ are a pair of orthogonal eigenstates, i.e. $\langle\mathbf{v} \mid \mathbf{u}\rangle=0$.

The correlation function of $A_{\xi}$ and $A_{\xi}^{2}$ can be calculated using Eq. (B10) once and twice respectively. The scalar products of $\langle\mathbf{v}|$ with the results of the commutation procedures are

$$
\left\langle\mathbf{v}\left|A_{\xi}\right| \mathbf{u}\right\rangle=-\sum_{x=1}^{N} a_{j} \frac{c_{\xi x}}{b_{\xi x}} \prod_{l=1 \neq j}^{N} \frac{1}{b_{x l}}\left\langle u_{x+1}, \xi, u_{x-1} \mid \mathbf{v}\right\rangle
$$

$$
\left\langle\mathbf{v}\left|A_{\xi}^{2}\right| \mathbf{u}\right\rangle=4 \sum_{x=1}^{N} a_{x} \frac{c_{\xi x}}{b_{\xi x}} \prod_{l ; l \neq x}^{N} \frac{1}{b_{x l}} \sum_{y ; y \neq x}^{N} a_{y} \frac{c_{\xi y}}{b_{\xi y}} \prod_{l ; l \neq x, y}^{N} \frac{1}{b_{x l}} \frac{1}{b_{y \xi}}\left\langle u_{x-1}, \xi, u_{x+1}, u_{y-1}, \xi, u_{y+1} \mid \mathbf{v}\right\rangle
$$

where the scalar products in the right hand sides are already given in Eqs. C11, C17) in explicit form.

Evaluation of the remaining $D_{\xi} A_{\xi}$ correlation function involves the same problem of taking the limit $v \rightarrow u=\xi$ in commutation relation Eq. (B9), as in the calculation of the -+ correlation function. Here we resolve it in the same 
way as we have already done in evaluating Eq. (C19). Expanding the numerator and the denominator of Eq. (B9) in $v-u \rightarrow 0$ we rewrite the $D_{\xi} A_{\xi}$ correlation function as

$$
\left\langle\mathbf{v}\left|D_{\xi} A_{\xi}\right| \mathbf{u}\right\rangle=\left\langle\mathbf{v}\left|\mathcal{T}_{\xi} A_{\xi}-A_{\xi}^{2}\right| \mathbf{u}\right\rangle-\sinh 2 \eta \lim _{\bar{\xi} \rightarrow \xi} \partial_{\bar{\xi}}\left\langle\mathbf{v}\left|C_{\xi} B_{\bar{\xi}}-C_{\bar{\xi}} B_{\xi}\right| \mathbf{u}\right\rangle
$$

We use the general result in Eq. (C9) and write the $C_{\xi} B_{\bar{\xi}}$ and $C_{\bar{\xi}} B_{\xi}$ correlation functions under the derivative as

$$
\begin{aligned}
\left\langle\mathbf{v}\left|C_{\bar{\xi}} B_{\xi}\right| \mathbf{u}\right\rangle= & \sum_{x=1}^{N} a_{x} \frac{c_{x \xi}}{b_{x \xi}} \prod_{i=1 \neq x}^{N} \frac{1}{b_{x i}} \sum_{y=1 \neq x}^{N} d_{y} \frac{c_{\xi y}}{b_{\xi y}} \prod_{j=1 \neq x, y}^{N} \frac{1}{b_{j y}}\left\langle u_{x-1}, \bar{\xi}, u_{x+1}, u_{y-1}, \xi, u_{y+1} \mid \mathbf{v}\right\rangle \\
& +\sum_{x=1}^{N} a_{x} \frac{c_{x \xi}}{b_{x \xi}} \prod_{i=1 \neq x}^{N} \frac{1}{b_{x i}} \prod_{j=1 \neq x}^{N} \frac{1}{b_{j \xi}}\left\langle u_{x-1}, \bar{\xi}, u_{x+1} \mid \mathbf{v}\right\rangle, \\
\left\langle\mathbf{v}\left|C_{\xi} B_{\bar{\xi}}\right| \mathbf{u}\right\rangle= & \sum_{x=1}^{N} a_{x} \frac{c_{x \bar{\xi}}}{b_{x \bar{\xi}}} \prod_{i=1 \neq x}^{N} \frac{1}{b_{x i}} \sum_{y=1 \neq x}^{N} d_{y} \frac{c_{\bar{\xi} y}}{b_{\bar{\xi} y}} \prod_{j=1 \neq x, y}^{N} \frac{1}{b_{j y}}\left\langle u_{x-1}, \xi, u_{x+1}, u_{y-1}, \bar{\xi}, u_{y+1} \mid \mathbf{v}\right\rangle \\
& +\sum_{x=1}^{N} a_{x} \frac{c_{x \bar{\xi}}}{b_{x \bar{\xi}}} \prod_{i=1 \neq x}^{N} \frac{1}{b_{x i}} \prod_{j=1 \neq x}^{N} \frac{1}{b_{j \bar{\xi}}}\left\langle u_{x-1}, \xi, u_{x+1} \mid \mathbf{v}\right\rangle .
\end{aligned}
$$

Then, taking the derivative over $\bar{\xi}$, the limit $\bar{\xi} \rightarrow \xi$, and substituting the pair of the expressions above in Eqs. C27, C30), together with the expressions in Eqs. (C28, C29), we obtain

$$
\begin{aligned}
G_{z z} & =\prod_{j}^{N} \frac{1}{b_{j \xi}} \sum_{j=1}^{N} a_{j} \frac{c_{\xi j}}{b_{\xi j}} \prod_{l=1 \neq j}^{N} \frac{1}{b_{j l}}\left\langle u_{j+1}, \xi, u_{j-1} \mid \mathbf{v}\right\rangle+\sum_{j=1}^{N} a_{j} \frac{c_{\xi j}}{b_{\xi j}} \prod_{l=1 \neq j}^{N} \frac{1}{b_{j l}} \sum_{j^{\prime}=1 \neq j}^{N} a_{j^{\prime}} \frac{c_{\xi j^{\prime}}}{b_{\xi j^{\prime}}} \prod_{l=1 \neq j, j^{\prime}}^{N} \frac{1}{b_{j^{\prime} l}} \\
& \times \frac{1}{b_{j^{\prime} \xi}}\left\langle u_{j-1}, \xi, u_{j+1}, u_{j^{\prime}-1}, \xi, u_{j^{\prime}+1} \mid \mathbf{v}\right\rangle+\frac{\sinh 2 \eta}{2}\left[\sum_{x=1}^{N} a_{x} \frac{c_{x \xi}}{b_{x \xi}} \prod_{i=1 \neq x}^{N} \frac{1}{b_{x i}} \sum_{y=1 \neq x}^{N} \frac{c_{\xi y}}{b_{\xi y}}\right. \\
& \times\left(\tanh \left(u_{x}-\eta\right)+\tanh \left(u_{y}-\eta\right)\right) \prod_{j=1 \neq x, y}^{N} \frac{1}{b_{j y}}\left\langle u_{x-1}, \xi, u_{x+1}, u_{y-1}, \xi, u_{y+1} \mid \mathbf{v}\right\rangle \\
& +\sum_{x=1}^{N} a_{x} \frac{c_{x \xi}}{b_{x \xi}} \prod_{i=1 \neq x}^{N} \frac{1}{b_{x i}} \prod_{j=1 \neq x}^{N} \frac{1}{b_{j \xi}}\left[\tanh \left(u_{x}-\eta\right)+\tanh \left(v_{x}-\eta\right)\right. \\
& +\sum_{j^{\prime}=1 \neq x}^{N}\left[\tanh \left(v_{j^{\prime}}-\eta\right)-\tanh \left(u_{j^{\prime}}+\eta\right)\right]\left\langle u_{x-1}, \xi, u_{x+1} \mid \mathbf{v}\right\rangle \\
& \left.-\sum_{x=1}^{N} a_{x} \frac{c_{x \xi}}{b_{x \xi}} \prod_{i=1 \neq x}^{N} \frac{1}{b_{x i}} \prod_{j=1 \neq x} \frac{1}{b_{j \xi}}\left\langle u_{x-1}, \xi, u_{x+1} \mid \mathbf{v}\right\rangle^{\prime}\right]
\end{aligned}
$$

where all scalar products are already given in explicit form above.

Finally, we substitute Eqs. (C10, C23, C33) in Eq. (C1), rewrite the result in a more compact form by collecting similar terms, and use a general matrix identity $\operatorname{det} \hat{T}+\sum_{x=1}^{N} \operatorname{det} \hat{T}^{(x)}=\operatorname{det}(\hat{T}+\hat{X})$, where the matrix $T^{(b)}$ is obtained by substitution of the $x^{\text {th }}$ column from the matrix $\hat{X}$ and rank of $\hat{X}$ is equal to one. After constructing the corresponding matrices $\hat{X}$ for a single sum over $x$ and for a sum over only one variable in the double sum over $x, y$ we obtain

$$
\begin{aligned}
& \left\langle\mathbf{q}\left|\mathbf{S}_{1} \cdot \mathbf{S}_{2}\right| 0\right\rangle=\frac{\prod_{j}^{N} \cosh \left(v_{j}+\eta\right)}{\prod_{i<j} \sinh \left(v_{i}-v_{j}\right)} \sum_{x=1}^{N}(-1)^{x} \frac{\prod_{i, j ; j \neq x}^{N} \sinh \left(u_{j}-v_{i}\right)}{\prod_{j}^{N} \cosh ^{2}\left(u_{j}-\eta\right)} \prod_{l=1 \neq x}^{N} \frac{\sinh \left(u_{l}-u_{x}+2 \eta\right)}{\sinh \left(u_{l}-u_{x}\right)}\left[\operatorname{det} \hat{K}^{(x)}\right. \\
& \left.-\left(1-\frac{\sinh (2 \eta) \sinh \eta \sinh u_{x} \prod_{j ; j \neq x}^{N} \cosh \left(u_{j}+\eta\right)}{\prod_{i<j \neq x} \sinh \left(u_{i}-u_{j}\right)}\right) \operatorname{det} \hat{G}^{(x)}\right]-\frac{\prod_{j} \cosh \left(u_{j}+\eta\right) \prod_{j} \cosh \left(v_{j}+\eta\right)}{\prod_{j} \cosh ^{2}\left(u_{j}-\eta\right) \prod_{i<j} \sinh \left(v_{i}-v_{j}\right)} \operatorname{det} \hat{K}
\end{aligned}
$$


where the matrix elements are

$$
\begin{gathered}
K_{a b}=T_{a b}+(-1)^{b} \frac{\sinh ^{3}(2 \eta) \sinh \eta \sinh u_{b}}{\cosh \left(u_{b}+\eta\right)} \frac{\prod_{j, i \neq b}^{N} \sinh \left(u_{i}-v_{j}\right)}{\prod_{i<j \neq b} \sinh \left(u_{i}-u_{j}\right)} \prod_{l=1 \neq b}^{N} \frac{\sinh \left(u_{l}-u_{b}+2 \eta\right)}{\sinh \left(u_{l}-u_{b}\right)} \\
\times \frac{\frac{\sinh u_{b}}{\cosh \left(u_{b}+\eta\right) \cosh \eta}+\sum_{l=1}^{N}\left[\tanh \left(v_{l}+\eta\right)-\tanh \left(u_{l}+\eta\right)\right]}{\cosh \left(v_{a}-\eta\right) \cosh \left(v_{a}+\eta\right)}, \\
T_{a b}=\frac{\cosh ^{L}\left(v_{b}-\eta\right)}{\cosh ^{L}\left(v_{b}+\eta\right)} \frac{\sinh (2 \eta)}{\sinh ^{2}\left(v_{b}-u_{a}\right)} \prod_{j=1 \neq a}^{N} \frac{\sinh \left(v_{b}-u_{j}+2 \eta\right)}{\sinh \left(v_{b}-u_{j}\right)}-\frac{\sinh (2 \eta)}{\sinh ^{2}\left(u_{a}-v_{b}\right)} \prod_{j=1 \neq a}^{N} \frac{\sinh \left(u_{j}-v_{b}+2 \eta\right)}{\sinh \left(u_{j}-v_{b}\right)}, \\
K_{a b}^{(x)}=T_{a b}+\frac{(-1)^{b} \sinh ^{3}(2 \eta) \operatorname{sgn}(x-b)}{\cosh \left(v_{a}-\eta\right) \cosh \left(v_{a}+\eta\right)} \prod_{l=1 \neq x, b}^{N} \frac{\sinh \left(u_{l}-u_{b}+2 \eta\right)}{\sinh \left(u_{l}-u_{b}\right)} \frac{\cosh \left(u_{b}+\eta\right) \cosh \left(u_{x}-\eta\right)}{\prod_{i}^{N} \sinh \left(u_{b}-v_{i}\right) \prod_{i<j \neq x, b} \sinh \left(u_{i}-u_{j}\right)} \\
\left(\frac{\cosh \left(u_{b}-\eta\right)}{\cosh \left(u_{b}+\eta\right)}-\frac{\sinh \left(u_{x}-u_{b}+2 \eta\right)}{\sinh \left(u_{x}-u_{b}-2 \eta\right)}+\frac{\sinh 2 \eta \cosh \left(u_{b}-2 \eta\right) \sinh u_{x}}{\cosh \left(u_{x}-\eta\right) \cosh \left(u_{b}+\eta\right)}, \quad\right.
\end{gathered}
$$

when $b \neq x$,

$$
K_{a x}^{(x)}=\frac{\sinh (2 \eta) \sinh \left(2 v_{a}\right)}{\cosh ^{2}\left(v_{a}-\eta\right) \cosh ^{2}\left(v_{a}+\eta\right)}
$$

when $b=x, G_{a b}^{(x)}=T_{a b}$ when $b \neq x$, and $G_{a x}^{(x)}=K_{a x}^{(x)}$ when $b=x$. The result in Eq. (C34) was checked numer- ically for $N=2,3$ using the direct summation over the spacial coordinates. Eqs. (C34 C37) are Eqs. (13,16).
* present address: Dresden High Magnetic Field Laboratory, Helmholtz-Zentrum Dresden-Rossendorf, D-01314 Dresden, Germany.

$\dagger$ present address: Laboratory for Neutron Scattering and Imaging, Paul Scherrer Institute, 5232 Villigen, Switzerland.

1 B. Lüthi, Physical Acoustics in the Solid State (Springer, Berlin, 2005).

${ }^{2}$ K. Tani and H. Mori, Prog. Theor. Phys. 39, 876 (1968).

${ }^{3}$ K. Kawasaki and A. Ikushima, Phys. Rev. B 1, 3143 (1970).

4 Y. Zhou and P. A. Lee, Phys. Rev. Lett. 106, 056402 (2011).

5 M. Serbyn and P. A. Lee, Phys. Rev. B 87174424 (2013).

${ }^{6}$ T. Giamarchi, Quantum Physics in One Dimension (Clarendon Press, Oxford, 2003).

7 R. Coldea, D. A. Tennant, and Z. Tylczynski, Phys. Rev. B 68, 134424 (2003).

8 A. Sytcheva, O. Chiatti, J. Wosnitza, S. Zherlitsyn, A. A. Zvyagin, R. Coldea, and Z. Tylczynski, Phys. Rev. B 80, 224414 (2009).

${ }^{9}$ Y. Trudeau, M. Poirier, and A. Caillé, Phys. Rev. B 46, 169 (1992).

10 B. Lake, D. A. Tennant, C. D. Frost, and S. E. Nagler, Nat. Mater. 4, 329 (2005).

11 B. Wolf, S. Zherlitsyn, B. Lüthi, N. Harrison, U. Löw, V. Pashchenko, M. Lang, G. Margraf, H.-W. Lerner, E. Dahlmann, F. Ritter, W. Aßmus, and M. Wagner, Phys.
Rev. B 69, 092403 (2004).

12 M. Gaudin, The Bethe Wavefunction (Cambridge University Press, Cambridge, 2014).

13 O. Tsyplyatyev, A. J. Schofield, Y. Jin, M. Moreno, W. K. Tan, C. J. B. Ford, J. P. Griffiths, I. Farrer, G. A. C. Jones, and D. A. Ritchie, Phys. Rev. Lett. 114, 196401 (2015).

14 O. Tsyplyatyev, A. J. Schofield, Y. Jin, M. Moreno, W. K. Tan, A. S. Anirban, C. J. B. Ford, J. P. Griffiths, I. Farrer, G. A. C. Jones, and D. A. Ritchie, Phys. Rev. B 93, 075147 (2016).

15 M. Moreno, C. J. B. Ford, Y. Jin, J. P. Griffiths, I. Farrer, G. A. C. Jones, D. A. Ritchie, O. Tsyplyatyev, and A. J. Schofield, Nat. Commun. 7, 12784 (2016).

16 N. Kitanine, J. M. Maillet, V. Tetras, Nucl. Phys. B 554, 647 (1999).

17 N. Kitanine, J. M. Maillet, Nucl. Phys. B 567, 554 (2000).

18 J.-S. Caux and J. M. Maillet, Phys. Rev. Lett. 95, 077201 (2005).

19 J.-S. Caux, P. Calabrese, and N. Slavnov, J. Stat. Mech. 2007, P01008 (2007).

20 B. Lake, D. A. Tennant, J.-S. Caux, T. Barthel, U. Schollwöck, S. E. Nagler, and C. D. Frost, Phys. Rev. Lett. 111, 137205 (2013).

21 A Kamra and G. E. W. Bauer, Solid State Commun. 198, 35 (2014).

22 A. Kamra, H. Keshtgar, P. Yan, and G. E. W. Bauer, Phys. Rev. B 91, 104409 (2015).

23 A. Rückriegel, P. Kopietz, D. A. Bozhko, A. A. Serga, and 
B. Hillebrands, Phys. Rev. B 89, 184413 (2014).

24 A. Rückriegel and P. Kopietz, Phys. Rev. Lett. 115, 157203 (2015).

25 R. Coldea, D. A. Tennant, R. A. Cowley, D. F. McMorrow, B. Dorner, and Z. Tylczynski, Phys. Rev. Lett. 79, 151 (1997).

26 M. Kohno, O. A. Starykh, and L. Balents, Nat. Phys. 3, 790 (2007).

27 M. T. Hutchings, G. Shirane, R. J. Birgeneau, and S. L. Holt, Phys. Rev. B 5, 1999 (1972).

28 O. M. Auslaender, A. Yacoby, R. de Picciotto, K. W. Baldwin, L. N. Pfeiffer, and K. W. West, Science 295, 825 (2002).

29 Y. Jompol, C. J. B. Ford, J. P. Griffiths, I. Farrer, G. A. C. Jones, D. Anderson, D. A. Ritchie, T. W. Silk, A. J. Schofield, Science 325, 597 (2009).

30 Complex quasimomenta (string solutions) at the $\Delta=1$ point emerge under the numerical deformation, starting from the real quasimomenta at the $\Delta=0$ point.

31 R. Orbach, Phys. Rev. 112, 309 (1958).

${ }^{32}$ V. E. Korepin, N. M. Bogoliubov, A. G. Izergin, Quantum inverse scattering methods and correlation functions (Cambridge University Press, Cambridge, 1993).

33 M. Gaudin, B. M. McCoy, and T. T. Wu, Phys. Rev. D 23, 417 (1981).

34 V. E. Korepin, Commun. Math. Phys. 86, 391 (1982).

35 C. Kittel, Introduction to Solid State Physics (Wiley, New York, 1996).

36 A. Kreisel, P. Kopietz, P. T. Cong, B. Wolf, and M. Lang, Phys. Rev. B 84, 024414 (2011).

37 S. Streib, P. Kopietz, P. T. Cong, B. Wolf, M. Lang, N. van Well, F. Ritter, and W. Aßmus, Phys. Rev. B 91, 041108(R) (2015).
38 The width of the anti-crossing is estimated as a matrix element of the magnetostrictive interaction in Eq. (3), $J_{1} \sqrt{\hbar b /\left(m \omega_{D}\right)} / L^{4}$ (where the spin matrix element belongs the second level of the hierarchy $\sim 1 / L^{4}$ ) times the superradiant enhancement factor $\left(\omega_{D} L / J\right)^{3 / 2}$ since the number of the almost degenerate levels is $\sim\left(\omega_{D} L / J\right)^{3}$.

39 N. Krüger, S. Belz, F. Schossau, A. A. Haghighirad, P. T. Cong, B. Wolf, S. Gottlieb-Schoenmeyer, F. Ritter, and W. Aßmus, Cryst. Growth Design 10, 4456 (2010).

40 B. Lüthi, G. Bruls, P. Thalmeier, B. Wolf, D. Finsterbusch, and I. Kouroudis, J. Low Temp. Phys. 95, 257 (1994).

41 R. Truell, C. Elbaum, and B. B. Chick, Ultrasonic methods in solid state physics (Academic Press, New York, 1969).

42 P. T. Cong, L. Postulka, B. Wolf, N. van Well, F. Ritter, W. Aßmus, C. Krellner, M. Lang, J. Appl. Phys. 120, 142113 (2016).

43 L. Balents, Nature 464, 199 (2010).

${ }^{44}$ In this paper we assume that $N$ is even obtaining $q_{j}=$ $2 \pi\left(I_{j}+1 / 2\right) / L$ in the $\Delta \rightarrow 0$ limit. For odd $N$ s the $\Delta \rightarrow 0$ limit of Eqs. (A2, A3) gives $q_{j}=2 \pi I_{j} / L$ that is a parity effect. We do not consider it here.

45 E. H. Lieb, T. D. Schultz, and D. C. Mattis, Ann. Phys. (N.Y.) 16, 407 (1961).

46 J. D. Noh, D.-S. Lee, and D. Kim, Physica A 287, 167 (2000).

47 K. Fabricius and B. M. McCoy, J. Stat. Phys. 103, 647 (2001).

48 F. H. L. Essler, V. E. Korepin, and K. Schoutens, J. Phys. A 25, 4115 (1992).

49 R. J. Baxter, J. Stat. Phys. 108, 1 (2002).

50 N. A. Slavnov, Theor. Math. Phys. 79, 502 (1989).

51 J. M. Maillet and J. Sanchez de Santos, Amer. Math. Soc. Transl. 201(2), 137 (2000), arXiv:q-alg/9612012 